\title{
Large deformations and fluorescence response of mechanochromic polyurethane sensors
}

\author{
Filippo Cellini ${ }^{\mathrm{a}}$, Liwei Zhou ${ }^{\mathrm{a}}$, Sachin Khaplib ${ }^{\mathrm{b}}$, Sean D. Peterson ${ }^{\mathrm{a}, \mathrm{c}}$, Maurizio Porfiri ${ }^{\mathrm{a}, *}$ \\ ${ }^{a}$ Department of Mechanical and Aerospace Engineering, Tandon School of Engineering, \\ New York University, Six MetroTech Center, Brooklyn, NY 11201, USA \\ ${ }^{b}$ Engineering Division, New York University Abu Dhabi, Abu Dhabi 129188, UAE \\ ${ }^{c}$ Department of Mechanical and Mechatronics Engineering, University of Waterloo, Waterloo, Ontario N2L 3G1, Canada
}

\begin{abstract}
A mechanochromic composite comprises an excimer-forming fluorescent dye dispersed in a compatible polymeric host. Upon large deformation of the polymeric structure, a shift of the fluorescence emission from the excimer band to the monomer band is observed, due to reorganization of dye aggregates. Mechanochromic elastomers can be tailored to produce a reversible optical response during cyclic deformation. Here, we study the optomechanical properties of mechanochromic elastomers undergoing large uniaxial and biaxial deformations. Through detailed experiments and constitutive modeling, we relate the optical response of the mechanochromic elastomer to the deformation of the polymer network. We confirm that optical response is largely reversible and is modulated by the initial dye concentration in the polymer. We adapt the classical Arruda-Boyce model to elucidate microstructural modifications of the polymer-dye blend. We propose a constitutive hypothesis for the optical behavior of the material, which relates variations of the emission spectrum to surface stretching. This knowledge can aid in the design of strain and stress sensors for applications in mechanics, biomechanics, and life sciences.
\end{abstract}

Keywords: Fluorescent material, Mechanochromism, Polyurethane elastomers, Sensors, Stress relaxation

\section{INTRODUCTION}

The increasing interest of the scientific community in active materials that are able to convert mechanical stimuli into electrical or optical signals is fueled by their incredible number of applications in mechanics, biomechanics, and the life sciences (Cheng et al., 2012; Cohen Stuart et al., 2010; Ferrari et al., 2007; Harrison and Ounaies, 2002; Jo et al., 2013; Urban, 2011; Venugopal et al., 2014). Synthetic polymers are attractive to these fields of investigation for the possibility of tailoring their properties through modification of their chemical and physical structures (Debashish et al., 2010; Weder, 2011).

Several efforts have been devoted to the study of polymeric materials whose optical properties are modulated in response to mechanical deformation (Pucci and Ruggeri, 2011; Roberts and Holder, 2011). For example, mechanochromism has been demonstrated in soft polymeric materials embedding excimer-forming fluorescent molecules, where shifts in relative intensity of monomer to excimer emission are observed upon deformation of the polymer host and reorganization of dye aggregates (Crenshaw et al., 2007; Donati et al., 2008; Kunzelman et al., 2006; Pucci et al., 2005, 2011). Mechanochemical polymers present a detectable modification of their optical behavior upon rupture of a covalent bond from the stretching of polymer chains (Davis et al., 2009; May and Moore, 2013). More specifically, the rupture of a weak covalent bond in a chromogenic molecule induces reversible (Sottos, 2014) or irreversible (Kingsbury et al., 2011) shifts in the fluorescence emission of the material. Beyond fluorescent materials, light emission has been detected in

\footnotetext{
*Corresponding author. Tel.: +1-718-260-3681. Fax: +1-718-260-3532.

Email address: mporfiri@nyu.edu (Maurizio Porfiri)
} 
chemoluminescent materials upon activation of mechanochemical reactions (Chen and Sijbesma, 2014; Chen et al., 2012). Mechanochromic behavior has also been observed in soft photonic gels, where an applied stress induces a shift in the band gap of the photonic crystal, resulting in a variation of the spectrum of the reflected light (Chan et al., 2013; Foulger et al., 2001).

A fully reversible response is a highly desirable characteristic for mechanochromic systems, where we seek to obtain a complete recovery of the original optical properties as the deformation of the material is removed. Reversible optical response in a mechanochromic polymer has been obtained by blending crosslinked poly(cyclooctene) (PCO) with an oligo(p-phenylene vinylene) dye (Kunzelman et al., 2008), in which the shape memory behavior of the polymer has been leveraged to invert the process of dye reorganization in the polymeric matrix. Reversible mechanochromism has also been observed in polyurethane elastomers, either by substitution of oligo(p-phenylene vinylene) in the molecular structure (Crenshaw and Weder, 2006) or by dispersion of bis(benzoxazolyl)stilbene dye within the polymer network (Bao et al., 2013; Cellini et al., 2014). Reversible response to mechanical stress has been recently demonstrated for mechanochemical polymers by embedding a chromogenic spiropyran molecule in the cross-linked structure of a poly(dimethylsiloxane) polymer (Gossweiler et al., 2014). Moreover, successive mechanochemical activations of the chromogenic bond have been observed (Larsen and Boydston, 2014), and the effect of elastomer relaxation on mechanochemical response has been investigated (Beiermann et al., 2014).

Polyurethane elastomers are attractive for the design of polymer-dye blends with reversible optical response (Bao et al., 2013; Cellini et al., 2014). These materials can sustain large deformations with an almost complete recovery of the original shape (Wang and Pinnavaia, 1998), enabling the cancellation of optomechanical modifications (Gossweiler et al., 2014; Kunzelman et al., 2008). Polyurethane elastomers are two phase systems, with a hard phase composed of diisocyanates dispersed in a soft phase network of polyol chains (Desai et al., 2000; Petrovic and Ferguson, 1991; Petrovic et al., 1998). Similar to filled rubbers (Bergstrom and Boyce, 1999), foams (Gupta et al., 2013; Rizzi et al., 2000; Thiyagasundaram et al., 2010), and gels (Deng and Pence, 2010; Wallmersperger et al., 2013), the structural morphology of polyurethane elastomers evolves during mechanical loading (Qi and Boyce, 2005). Shear stresses induced by chain stretching and the evolution of the material morphology favor the reorganization of dye aggregates, which is responsible for variations in the optical response of the polymer. The latter constitutes a mechanism that has been previously associated with mechanochromic response in different polymer-dye blends (Crenshaw et al., 2007; Donati et al., 2008; Kunzelman et al., 2006; Pucci et al., 2005). Furthermore, polyurethane elastomers are bio-compatible materials (Feng and Ye, 2011; Stokes et al., 1995), affording potential applications in the environmental and life sciences.

In this study, we investigate reversible mechanochromic behavior of thermoplastic polyurethane elastomers blended with bis(benzoxazolyl)stilbene dyes (TPU-BBS). Material response for large deformations under uniaxial and biaxial extensions is studied to elucidate the relationship between optical response and stretching of the elastomeric network, microstructural evolution of the polymer, and dye concentration. Salient mechanical and optical properties of mechanochromic TPU-BBS elastomers are identified from uniaxial stress-relaxation experiments. Samples are subject to complete loading-unloading cycles, composed of successive steps where the film is slowly stretched and then relaxes toward the equilibrium. This allows for the separation of time-dependent viscoelastic effects from time-independent chain stretching in the constitutive response of the polymer (Cellini et al., 2014; Qi and Boyce, 2005). To assess mechanochromic behavior in a more complex loading condition, mechanical stretch and fluorescence emission are measured during biaxial extension experiments. More specifically, we study the inflation-deflation cycle of a membrane, which is executed in a continuous one-step process to avoid creep during constant pressurization of the membrane. Mechanical testing of membranes has been often used to study polymeric (Selvadurai and Shi, 2012) and biological materials (Gambarotta et al., 2005).

Experimental results are interpreted through a modified form of the Arruda-Boyce model (Arruda and Boyce, 1993), which accounts for the evolution of the microstructure during deformation (Qi and Boyce, 2004, 2005). To model the optical response during deformation of the material, we propose a new constitutive hypothesis, which relates the fluorescence emission to the surface stretching of the elastomer. This constitutive relationship is calibrated on experimental data using a single parameter that depends on the initial concentration of the dye. 

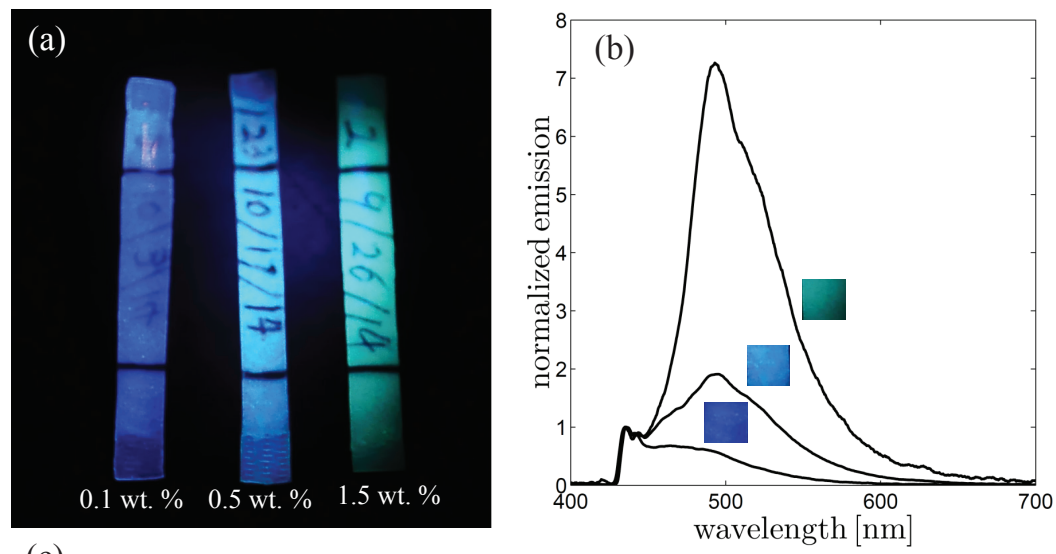

(c)

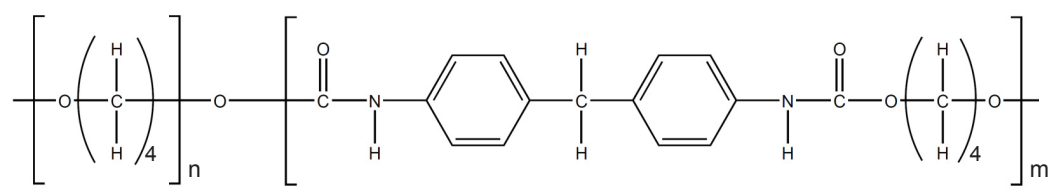

(d)

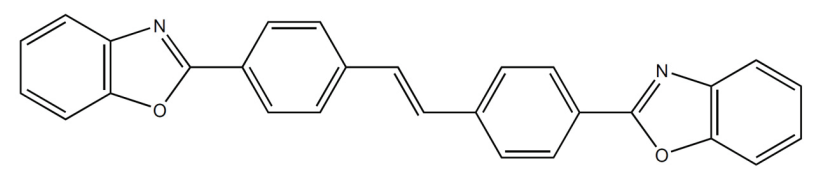

Figure 1: (a) Photographic image of three TPU-BBS samples with concentrations $0.1,0.5$, and 1.5 wt.\%. The image is obtained through a commercial linear plastic polarizer. (b) Fluorescence spectra for the three concentrations are reported in the graph. Spectra are normalized so that the emission is equal in correspondence of the monomer peak at $436 \mathrm{~nm}$. The fluorescence emission of dye aggregates increases with dye concentration and is associated with the progressive shift of material coloration from blue to green. (c) Chemical structure of thermoplastic polyurethane elastomer adapted from Bao et al. (2013). (d) Chemical structure of the bis(benzoxazolyl)stilbene molecule.

The paper is organized as follows. In Section 2, we present a description of the experimental apparatus for optomechanical testing, and we introduce the constitutive model used for the analysis. Experimental results are reported and discussed in Section 3, while conclusions are summarized in Section 4. Appendix A succinctly describes the model used to interpret the results of the inflation test.

\section{Materials and methods}

\subsection{Fabrication of mechanochromic materials}

Mechanochromic polymer-dye blends are thermoplastic polyurethane (TPU ESTANE - 90AE purchased from Lubrizol) blended with $0.1,0.5$, and $1.5 \mathrm{wt}$ \% of bis(benzoxazolyl)stilbene (BBS) fluorescent dye (Sigma Aldrich). Polymeric films with uniform thicknesses in the range $0.2-0.3 \mathrm{~mm}$ are obtained via solution casting following a standard procedure for fabrication of mechanochromic composites (Ciardelli et al., 2013). The dye is dispersed in dimethylformamide (99.8\% purity, Sigma-Aldrich) and sonicated for 10 minutes. Solventdye solutions are prepared with dye concentrations of $0.1,0.5$, and $1.5 \mathrm{wt} . \%$. The solvent-dye mixture is added to the polymer, which is dissolved by vigorous stirring at $90^{\circ} \mathrm{C}$ for two hours. The solution is cast in a metallic mold and left to dry overnight at $60^{\circ} \mathrm{C}$. In Figure $1(\mathrm{a})$ and (b), we display images of films with three different concentrations taken using a Nikon D90 CMOS camera (12.3 Mpixel) and a linear plastic polarizer (P/N 85920, www.edmundoptics.com) together with the corresponding fluorescence emission. Chemical structures of the polymer (TPU) and dye molecules (BBS) are reported in Figure 1(c) and (d).

Emission spectra are recorded using an Ocean Optics USB2000+ portable spectrometer connected to an optical fiber probe with numerical aperture (NA) 0.22 (www.oceanoptics.com). The UV source is composed 
(a)

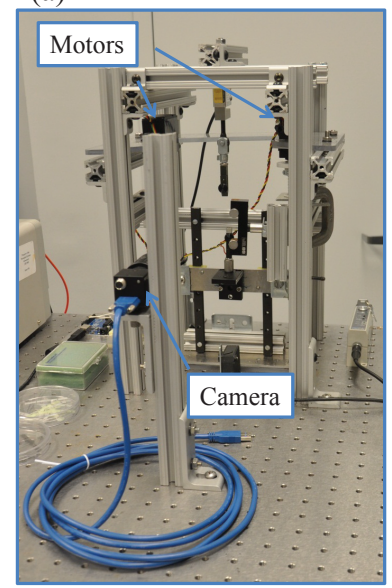

(b)

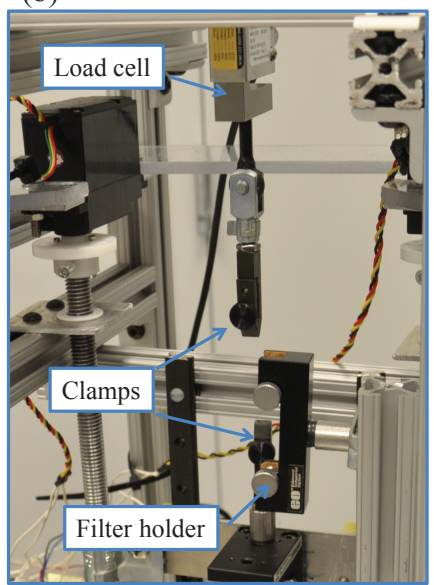

Figure 2: (a) Experimental apparatus for uniaxial stress investigations assembled on an optical table. (b) Close-up view of the clamping system.

of light emitting diodes with emission centered at $382.5-385 \mathrm{~nm}$. The excitation band is filtered before acquisition of the spectrum through a long-pass fluorescence dichroic filter at $409 \mathrm{~nm}$ (www.edmundoptics.com). Spectra are corrected by subtracting the dark background, acquired at the beginning of the experiment. The increased concentration of dye in the polymer is responsible for the shift of the visible coloration from blue to green, see Figure 1(b), which is associated with the increased contribution of dye aggregates to the spectrum of the excimer emission in the band $470-550 \mathrm{~nm}$. In the solid state, the formation of BBS aggregates is likely favored by the planar molecule conformation, where aggregated structures present sandwich-type conformations with interplanar distances of a few Angstroms (Pucci et al., 2007). Fluorescence emission of dye aggregates occurs at a lower energy with respect to the emission of the single molecule (Makowski et al., 2011). This lower energy level corresponds to a larger wavelength in the emitted light and a shift of the coloration in the visible spectrum from blue (monomer) to green (excimer), see Figure 1(b).

\subsection{Uniaxial loading}

Stress-relaxation experiments are conducted on a custom-made miniature experimental apparatus whose main features are presented in Cellini et al. (2014). The experimental apparatus assembled on an optical table is displayed in Figure 2(a). Experiments are designed to simultaneously collect force, deformation, and fluorescence emission spectrum caused by irradiation through a narrow-band UV source.

TPU-BBS films are cut into samples of area $48 \times 5 \mathrm{~mm}^{2}$. The shorter edges of the samples are clamped for a $6 \mathrm{~mm}$ length to the apparatus and a uniaxial force is applied by two SC- 8 Imada gauge force attachments. The clamp positions are controlled through two servo motors, and the applied load is measured using a FUTEK LSB303 25 lb S-beam type load cell (www.futek.com) connected to a NI USB-6221 data acquisition board (www.ni.com), see Figure 2(b). The sample deformation is recorded using a FLEA FL3-U3-13E4C-C camera with acquisition frequency set to 15 frames per second (fps). To minimize the effect of the clamps on the stretch measurement, we track two fiduciary marks placed $6 \mathrm{~mm}$ from the edges of the clamps, yielding a gauge length of $24 \mathrm{~mm}$. Tracking is implemented during image postprocessing via the feature tracking technique in the Proanalyst software package (www.xcitex.com).

Spectra are recorded using the Ocean Optics USB2000+ spectrometer, filtering the emitted light before acquisition via a long-pass fluorescence dichroic filter at $409 \mathrm{~nm}$. The integration time for the photon count process is set to $3 \mathrm{~s}$. The intensity of light emission in the monomer and excimer bands $\mathcal{I}$ is determined by separately fitting the two peaks with a four parameters Lorentzian function

$$
\mathcal{I}\left(\lambda_{\mathrm{w}}\right)=\frac{a_{1}}{\left(\left(\lambda_{\mathrm{w}}-a_{2}\right)^{2}+a_{3}\right)}+a_{4}
$$


(a)

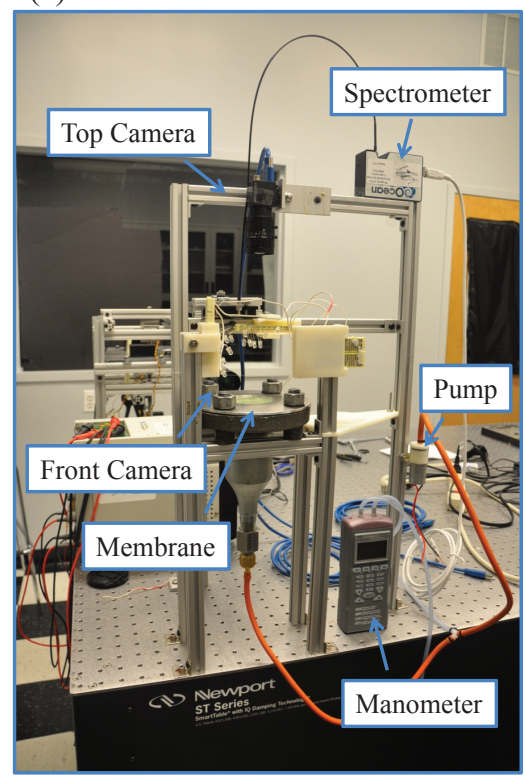

(b)

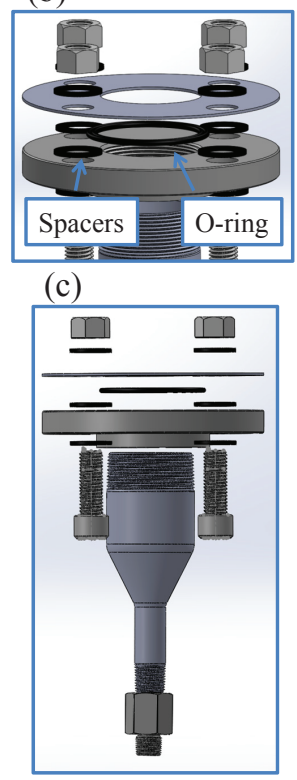

Figure 3: (a) Experimental apparatus for inflation of a mechanochromic membrane. (b) Close-up view of the membrane clamp on the pressure chamber. (c) Exploded view of the pressure chamber assembly.

where $\lambda_{\mathrm{w}}$ is the wavelength of the emitted light and $a_{1}, a_{2}, a_{3}$ and $a_{4}$ are fitting parameters determined using the least squares curve fitting routine in Matlab (www.mathworks.com). The effective intensity of the peak is determined as the maximum of the fitted curve. The wavelength range used to fit the monomer emission peak is from 427 to $445 \mathrm{~nm}$ for concentrations 0.1 and $0.5 \mathrm{wt}$. \% and from 427 to $441 \mathrm{~nm}$ for $1.5 \mathrm{wt}$. \%. Fitting is performed by using a minimum of 40 experimental points to identify the parameters of the function in equation (1). The wavelength interval employed in the fitting of the excimer emission peak ranges from 454 to $561 \mathrm{~nm}$ for concentrations 0.1 and $0.5 \mathrm{wt} . \%$ and from 460 to $509 \mathrm{~nm}$ for $1.5 \mathrm{wt} \%$, wherein a minimum of 130 experimental points are used in the fitting.

Following Cellini et al. (2014), uniaxial relaxation tests are conducted on the samples with the strain rate set to $0.025 \mathrm{~s}^{-1}$. The loading is partitioned into successive small steps by controlling the distance between the clamps: each step consists of a $5 \mathrm{~s}$ linear stretch followed by a $60 \mathrm{~s}$ relaxation toward the local equilibrium. The sample is stretched up to $200 \%$ of the initial length before unloading begins. Similar to the loading phase, unloading consists of a $5 \mathrm{~s}$ linear stretch followed by $60 \mathrm{~s}$ relaxation until the applied load is completely released. To characterize the mechanochromic response over repeated loading cycles, we perform uniaxial loading of samples with concentration $0.5 \mathrm{wt} . \%$ for three consecutive loading cycles. During each cycle, samples are stretched up to $200 \%$ of their initial length, which is measured at the beginning of the test to account for residual deformation due to the loading history.

\subsection{Biaxial loading}

TPU-BBS membranes of diameter $80 \mathrm{~mm}$, nominal thickness $0.2-0.3 \mathrm{~mm}$, and $0.5 \mathrm{wt}$ \% dye concentration are cast using a cylindrical mold with a procedure similar to the one outlined in Section 2.1. Biaxial loading is applied to the TPU-BBS membranes through inflation experiments performed on the experimental apparatus shown in Figure 3(a). The apparatus affords controlled pressure loading of the membrane and simultaneous acquisition of deformation, internal pressure, and fluorescence emission. A schematic of the pressure chamber assembly is depicted in Figure 3(b) and (c). The pressure chamber is composed of a double threaded pipe with variable cross-section connected to a hose fitting adapter and a threaded flange on the top of the chamber. An EPDM o-ring is used to hold the membrane in position between the top flange and a $2 \mathrm{~mm}$-thick aluminum flange. Four bolts and plastic spacers are used to properly position the 
(a)

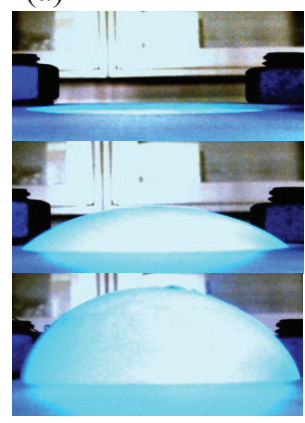

(b)

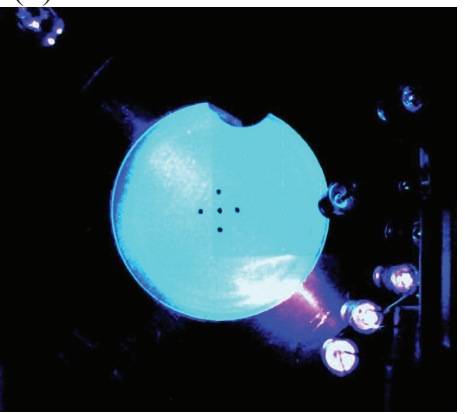

(c)

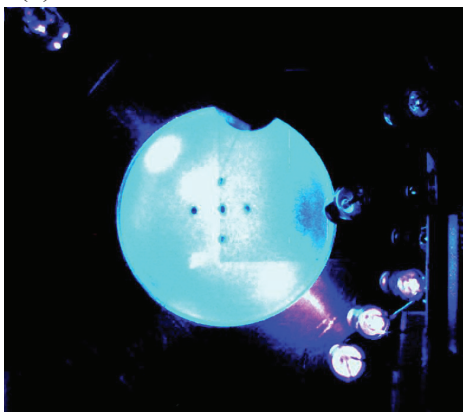

Figure 4: Frames captured from front (a) and top (b-c) cameras during inflation tests of a mechanochromic membrane with 0.5 wt.\% BBS in TPU. Colors displayed in this figure are different from colors displayed in Figure 1, since images are captured without the use of a polarizing filter.

aluminum flange. The testing diameter of the membrane after installation is $60 \mathrm{~mm}$, with a clamping length on the outer radius of the membrane of $10 \mathrm{~mm}$.

The chamber is pressurized through a $12 \mathrm{~V}$ mini air-pump connected to the chamber by rubber tubing. The internal pressure is monitored during the test by a Sper Scientific 840099 Digital Manometer, connected in parallel to the pump by a 3 -way hose connector. The deformation of the membrane is recorded from top and lateral views through two FLEA FL3-U3-13E4C-C cameras with acquisition frequencies set to 15 fps. Representative images recorded during the inflation test are displayed in Figure 4.

The membrane extension is measured using images recorded from the top view. More specifically, the biaxial deformation at the center of the membrane is determined by tracking 4 fiduciary marks positioned $5 \mathrm{~mm}$ from the center of the membrane. The marks are gently traced using a marker once the membrane is installed in the chamber. To guarantee correct and repeatable positioning of the marks, a custom-made drawing tool fitting the upper flange is employed. Side views are used to determine the height of the membrane center during the inflation test, with the position of the bottom flange used as a reference. Membrane height is measured using the one-dimensional edge detection tool in Proanalyst.

Fluorescence emission of the material is measured using the Ocean Optics USB2000+ spectrometer employed in the uniaxial loading tests. The probe is positioned at the center of the membrane, $100 \mathrm{~mm}$ from the surface, and a longpass filter with cut-on wavelength at $409 \mathrm{~nm}$ is used to filter out the excitation source, which is composed of 18 light emitting diodes $(382.5-385 \mathrm{~nm})$. Spectra are collected with $3 \mathrm{~s}$ integration times for the photon counting process over the entire duration of the test.

\subsection{Constitutive model}

Polyurethane elastomers are two phase systems composed of a soft phase, which is characterized by high chain mobility that contributes to the elastomeric behavior of the polymer network, and a hard phase, which is mainly responsible for the high elastic modulus (Petrovic and Ferguson, 1991; Petrovic et al., 1998). This microstructure may vary during large deformations, influencing the constitutive behavior of polyurethane elastomers, including their elastic moduli, viscoelastic responses, and hysteretic behaviors. Hysteresis is often found during large deformation cycles, characterized by progressive reductions of the effective modulus, often referred to as the "softening effect".

One approach to modeling the constitutive behavior of this class of materials is presented by Qi and Boyce (2005), where the softening effect is attributed to the progressive reduction of the hard phase and the corresponding increase in the volume fraction of the soft phase. Hard phase domains are assumed to act as rigid fillers in the soft phase elastomeric network. Under these assumptions, the macroscopic behavior of polyurethane elastomers is assimilated to a filled elastomer, whose mechanical properties are determined by adhesion of polymer chains to hard fillers dispersed in the polymeric network. Here, we employ a modified form of the Arruda-Boyce model (Arruda and Boyce, 1993) for the study of filled elastomers 
initially presented by Qi and Boyce (2004), and specialized for thermoplastic polyurethane elastomers by Qi and Boyce (2005).

The behavior of the TPU-BBS blend is described by the constitutive relation (Qi and Boyce, 2005)

$$
\mathbf{T}=\frac{v_{\mathrm{s}} X \mu}{3} \frac{\sqrt{N}}{\Lambda_{\text {chain }}} \mathrm{L}^{-1}\left(\frac{\Lambda_{\text {chain }}}{\sqrt{N}}\right) \mathbf{F F}^{\mathrm{T}}-p \mathbf{I},
$$

where $\mathbf{T}$ is the Cauchy stress tensor, $\mathbf{F}$ is the deformation gradient, $p$ is the static pressure, and $\mathbf{I}$ is the identity tensor in three dimensions. The parameter $\mu$ is equal to $n k T$, where $n$ is the number of macromolecular chains per unit volume, $k$ is the Boltzmann constant, and $T$ is the absolute temperture. The function $\mathrm{L}^{-1}(x)$ is the inverse of the Langevin function $\mathrm{L}(x)=\operatorname{coth}(x)-\frac{1}{x}$, while $N$ is the number of statistical links in a chain between crosslinks, and $\Lambda_{\text {chain }}=\sqrt{X\left(\frac{J_{1}}{3}-1\right)+1}$ is the chain extension, with $J_{1}$ being the first invariant of $\mathbf{F F}^{\mathrm{T}}$. The effect of the evolving microstructure is described through the volume fraction of the soft phase $v_{\mathrm{s}}$ and the stretch amplification factor $X$, which accounts for the presence of the hard phase that acts as a rigid filler in the soft phase network.

The evolution of the microstructure of the polymer network is described through (Qi and Boyce, 2004)

$$
\dot{v}_{\mathrm{s}}=A\left(v_{\mathrm{ss}}-v_{\mathrm{s}}\right) \frac{\sqrt{N}-1}{\left(\sqrt{N}-\Lambda_{\text {chain }}^{\max }\right)^{2}} \dot{\Lambda}_{\text {chain }}^{\max },
$$

where a superimposed dot indicates differentiation with respect to time, the parameter $A$ regulates the rate of variation, $\Lambda_{\text {chain }}^{\max }$ is the maximum chain extension during the previous loading history, and $v_{\mathrm{ss}}$ is the upper limit of $v_{\mathrm{s}}$. We assume damage of the material to be permanent and the original composition of the microstructure not to be recovered as the material returns to its original configuration. We introduce the constraint

$$
\dot{\Lambda}_{\text {chain }}^{\max }= \begin{cases}0, & \Lambda_{\text {chain }}<\Lambda_{\text {chain }}^{\max } \\ \dot{\Lambda}_{\text {chain }}, & \Lambda_{\text {chain }} \geq \Lambda_{\text {chain }}^{\text {max }}\end{cases}
$$

to account for the permanent conversion of the soft phase. The evolution mechanism is active only if $\Lambda_{\text {chain }} \geq \Lambda_{\text {chain }}^{\text {max }}$, that is, when the network stretch exceeds the maximum stretch experienced by the material during the loading history. If such condition is not satisfied, the material microstructure does not change,

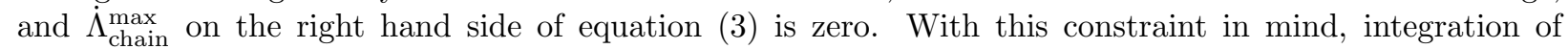
equation (3) yields

$$
v_{\mathrm{s}}= \begin{cases}\text { const }, & \Lambda_{\text {chain }}<\Lambda_{\text {chain }}^{\max } \\ v_{\mathrm{ss}}-\left(v_{\mathrm{ss}}-v_{\mathrm{s} 0}\right) \exp \left(-A \frac{\Lambda_{\text {chain }}-1}{\sqrt{N}-\Lambda_{\text {chain }}}\right), & \Lambda_{\text {chain }} \geq \Lambda_{\text {chain }}^{\text {max }}\end{cases}
$$

where $v_{\mathrm{s} 0}$ is the volume fraction of the soft phase in the virgin material. Thus, the volume fraction of soft phase $v_{\mathrm{s}}$ increases with the chain extension $\Lambda_{\text {chain. }}$. We note that $v_{\mathrm{s}}$ and $\Lambda_{\text {chain }}^{\max }$ never decrease, as they are both associated with permanent damage of the material due to the maximum chain stretch in the loading history. Thus, equation (5) effectively represents a damage accumulation and, as such, is valid for all loading/unloading cycles.

The stretch amplification factor $X$ is a function of the volume fraction of the hard phase (Bergstrom and Boyce, 1999). The effect of the rigid filler suspension on the elastic modulus of elastomeric materials has been investigated by several authors (Bergstrom and Boyce, 2000; Castaneda, 1989; Govindjee and Simo, 1991; Horgan et al., 2004). The model presented by Guth (1945) accounts for the effect of filler particle cohesion for elastomers with volume fractions of the rigid phase over $10 \%$. We hypothesize that the stiffening effect of the hard phase fillers in TPU-BBS blends is described by the following phenomenological law (Guth, 1945) for the stretch amplification factor:

$$
X=1+0.67 f\left(1-v_{\mathrm{s}}\right)+1.62 f^{2}\left(1-v_{\mathrm{s}}\right)^{2},
$$

where $\left(1-v_{\mathrm{s}}\right)$ is the volume fraction of the hard phase and $f$ is a shape factor, which accounts for the effect of cohesion of hard phase fillers and is constant during the microstructure evolution. This phenomenological 
law is different from the relation adopted in Cellini et al. (2014) to predict the constitutive behavior of TPU-BBS blends, whereby it introduces the additional parameter $f$ to capture the high concentration of hard phase fillers in the soft matrix. The stretch amplification factor can be determined as a function of the volume fraction $v_{\mathrm{s}}$ and the chain extension $\Lambda_{\text {chain }}$ by simultaneously solving equations (5) and (6), with the constraint (4), and accounting for the definition of $\Lambda_{\text {chain }}$. The solution of this system of equations determines the microstructure as a function of the deformation history of the material, which in turn controls the parameters of the constitutive relation (2).

Fluorescence emission of TPU-BBS blends is controlled by the spatial configuration of the dye molecules in the polymer. During the deformation process, the state of aggregation, spatial distribution, and orientation of BBS molecules evolve due to the interaction of the dye molecular structures with the background polymer (Ciardelli et al., 2013; Makowski et al., 2011). In the TPU-BBS composite, the BBS molecules interact with the host polymer network through $\pi-\pi$ interactions with the aromatic rings and hydrogenbonding interactions with the polar carbamate groups in the polymer network (Bao et al., 2013). Because of these interactions, the reorganization of BBS molecules during stretching of the polymer network produces the observed mechanochromic response. More specifically, mechanochromic response can be attributed to the progressive disruption of dye aggregates during the deformation process (Bao et al., 2013; Pucci et al., 2005), while other studies have demonstrated that dichroism of fluorescent dye molecules, such as BBS, may also contribute to the modification of optical properties of polymer films (Pucci et al., 2006). Modifications at the molecular level result in a consistent variation of the fluorescence emission in the mechanochromic polymer, with a measurable shift of the emission from the band of the excited dye aggregates (or excimer emission, with intensity $\mathcal{I}_{\text {exc }}$ ) to the band of single molecules (or monomer emission, with intensity $\mathcal{I}_{\text {mon }}$ ).

We describe the mechanochromic behavior of the films in terms of the emission ratio $I=\mathcal{I}_{\text {mon }} / \mathcal{I}_{\text {exc }}$, and we assume the emission ratio is a function of the overall surface stretching. Specifically, we hypothesize that mechanochromic response can be described through the following linear relationship

$$
I-I_{0}=\alpha\left(\frac{1}{|\mathcal{S}|} \int_{\mathcal{S}}\left\|\operatorname{det}(\mathbf{F}) \mathbf{F}^{-\mathrm{T}} \mathbf{N}\right\| \mathrm{d} A-1\right) .
$$

Here, $\left\|\operatorname{det}(\mathbf{F}) \mathbf{F}^{-\mathrm{T}} \mathbf{N}\right\|$ is the local stretch of a surface element $\mathrm{d} A$ with normal $\mathbf{N}$ in the reference configuration (Lacarbonara, 2013); $\mathcal{S}$ is the nominal outer surface of the film from which spectra are recorded during experiments; $I_{0}$ is the intensity ratio of the virgin material; and $\alpha$ quantifies the rate of variation of the emission during deformation. The parameters $I_{0}$ and $\alpha$ depend on initial concentration of the dye in the polymer, which controls the probability of dye molecules to form aggregates and, in turn, the fluorescence emission of the polymer.

Equation (7) assumes that the emission is only influenced by the surface of the film from where the emission is measured and that the overall change in surface area is the key geometric parameter that determines the film optical response. Thus, the present hypothesis does not take into consideration emission from the elastomer bulk and the opposite surface, which are likely to be relevant for thinner films (Shundo et al., 2012). Also, equation (7) posits that each portion of the outer surface equally contributes to the emission, which is expected to be valid for emission spectra recorded at a sufficient distance from the film (Hetch, 2002).

We specialize the constitutive relations (2) and (7) for the cases of uniaxial and biaxial extensions by assuming isochoric deformations $(\operatorname{det}(\mathbf{F})=1)$. For uniaxial extension, we set $\mathbf{F}=\operatorname{Diag}\{\lambda, 1 / \sqrt{\lambda}, 1 / \sqrt{\lambda}\}$, where $\lambda$ is the uniaxial stretch of the network. The stress-strain relation in equation (2) is determined solving for $p$ assuming that the stress normal to the loading direction is zero. Thus, we find that the axial stress $\sigma$ is given by

$$
\sigma=\frac{v_{\mathrm{s}} X \mu}{3} \frac{\sqrt{N}}{\Lambda_{\text {chain }}} \mathrm{L}^{-1}\left(\frac{\Lambda_{\text {chain }}}{\sqrt{N}}\right)\left(\lambda^{2}-\frac{1}{\lambda}\right),
$$

where $\Lambda_{\text {chain }}=\sqrt{X\left(\frac{1}{3}\left(\lambda^{2}+\frac{2}{\lambda}\right)-1\right)+1}$. The emission ratio for uniaxial extension is determined by substituting the expression for $\mathbf{F}$ into equation (7). By assuming an homogeneous state of deformation, equation (7) reduces to

$$
I-I_{0}=\alpha(\sqrt{\lambda}-1) .
$$


For the case of biaxial extension, we set $\mathbf{F}=\operatorname{Diag}\left\{\lambda_{1}, \lambda_{2}, 1 /\left(\lambda_{1} \lambda_{2}\right)\right\}$, where $\lambda_{1}, \lambda_{2}$ are the stretches in the principal directions along the plane of the undeformed membrane. The stress-strain relation is again determined by solving equation (2) for $p$ and assuming the stress through the thickness of the membrane to be zero. Thus, we find

$$
\begin{aligned}
& \sigma_{1}=\frac{v_{\mathrm{s}} X \mu}{3} \frac{\sqrt{N}}{\Lambda_{\text {chain }}} \mathrm{L}^{-1}\left(\frac{\Lambda_{\text {chain }}}{\sqrt{N}}\right)\left(\lambda_{1}^{2}-\frac{1}{\lambda_{1}^{2} \lambda_{2}^{2}}\right), \\
& \sigma_{2}=\frac{v_{\mathrm{s}} X \mu}{3} \frac{\sqrt{N}}{\Lambda_{\text {chain }}} \mathrm{L}^{-1}\left(\frac{\Lambda_{\text {chain }}}{\sqrt{N}}\right)\left(\lambda_{2}^{2}-\frac{1}{\lambda_{1}^{2} \lambda_{2}^{2}}\right),
\end{aligned}
$$

where $\sigma_{1}, \sigma_{2}$ are the stresses in the principal directions and the amplified chain stretch is $\Lambda_{\text {chain }}=$ $\sqrt{X\left(\frac{1}{3}\left(\lambda_{1}^{2}+\lambda_{2}^{2}+\frac{1}{\lambda_{1} \lambda_{2}}\right)-1\right)+1}$. For biaxial extension, the deformation is not homogeneous and equation (7) takes the form

$$
I-I_{0}=\alpha\left(\frac{1}{|\mathcal{S}|} \int_{\mathcal{S}} \lambda_{1} \lambda_{2} \mathrm{~d} A-1\right)
$$

\section{Results and Discussion}

\subsection{TPU-BBS elastomers exhibit mechanochromic response under uniaxial and biaxial cyclic loading}

The mechanochromic response of TPU-BBS elastomers is characterized through uniaxial testing of twenty-four virgin samples with dye concentrations $0.1,0.5$, and 1.5 wt. \% (eight samples per concentration). The nominal stress in the material $\frac{\sigma}{\lambda}$ is determined as the ratio of the force $F$ measured by the load cell and the initial cross sectional area $A_{0}$. The stretch $\lambda$ is computed as the ratio of the actual length $l$ to the initial gauge length $l_{0}$. The emission ratio $I$ is computed as the ratio between the intensity of the monomer emission $\mathcal{I}_{\text {mon }}$ and the excimer emission $\mathcal{I}_{\text {exc }}$, determined by separately fitting the monomer and excimer emission spectra using equation (1), as described in Section 2.2.

Values of the nominal stress, axial stretch, and emission ratio recorded during experiments for three samples with concentrations 0.1, 0.5, and $1.5 \mathrm{wt} . \%$ are displayed in Figure 5. The mechanical response of the three samples is comparable, and it is consistent with previous observations on polyurethane elastomers (Cellini et al., 2014; Qi and Boyce, 2005). As the film is initially stretched, a sharp increase of the stress is observed. During relaxation, the stress decreases, reaching an approximately constant value after $60 \mathrm{~s}$. During the unloading phase, the stress increases during relaxation after an initial decrease.

Emission ratios of the TPU-BBS elastomers exhibit a consistent increase with the polymer stretch along with a good degree of reversibility during the unloading phase, which is associated with the reduction of the polymer stretch and recovery of the initial shape. Figure 5 shows that mechanochromic response is equivalently exhibited during loading and unloading phases. Not only is the emission ratio recovered after a loading cycle, but the overall spectrum of the emission seems to be only marginally affected by the polymer relaxation. This is made evident from the spectra recorded during the experiments in Figure 6. For clarity, we normalize the spectra so that the emission is equal in correspondence of the monomer peak at $436 \mathrm{~nm}$. Figure 6(a) displays the fluorescence emission spectra at the beginning of the test and at the end of the loading phase for $\lambda \approx 2$. Similarly, Figure $6(\mathrm{~b})$ illustrates the spectra at the beginning of the unloading phase for $\lambda \approx 2$ and at the end of the test. A comparison of Figure 6(a) and (b) suggests a good degree of reversibility of the mechanochromic response of TPU-BBS blends, with an almost complete recovery of the entire emission spectrum after a loading cycle. Comparing emission spectra at the beginning and at the end of the test, we observe a small reduction of the excimer emission at the end of the loading cycle, which can be related to a permanent conversion of part of the dye aggregates to the monomeric phase after stress recovery.

Similar behavior is observed during biaxial extension of TPU-BBS membranes. Figure 7 displays the internal pressure $P$ per unit thickness $h$, the stretch at the center of the membrane $\lambda$, and the emission ratio as a function of time for a membrane experiment. To compare experiments with the uniaxial case, we define an equivalent strain rate as the ratio between the maximum strain and the time elapsed from the 

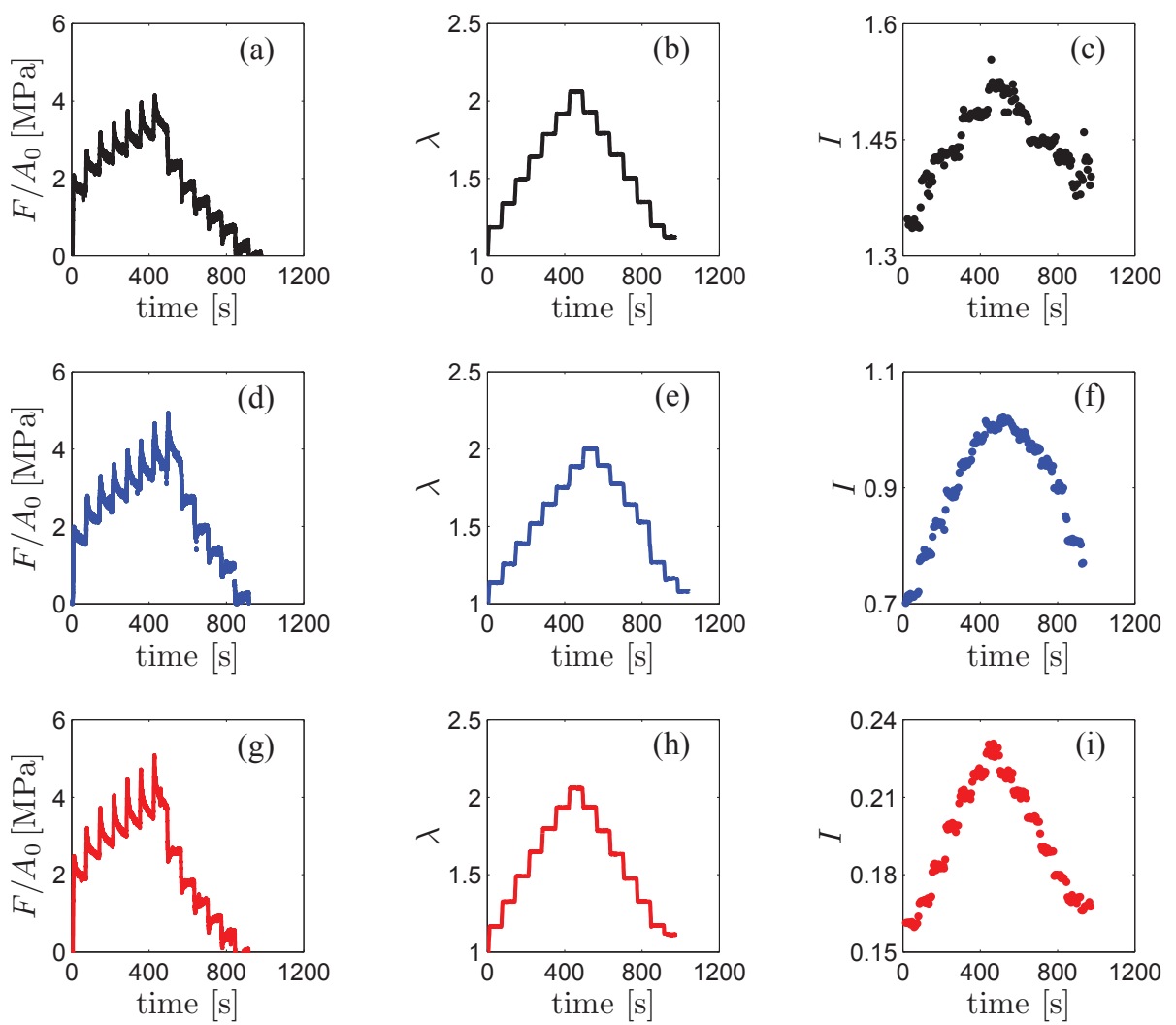

Figure 5: Nominal stress, stretch, and emission ratio as a function of time for three samples with dye concentration (a-c) 0.1, (d-f) 0.5 , and $(g-i) 1.5$ wt. $\%$
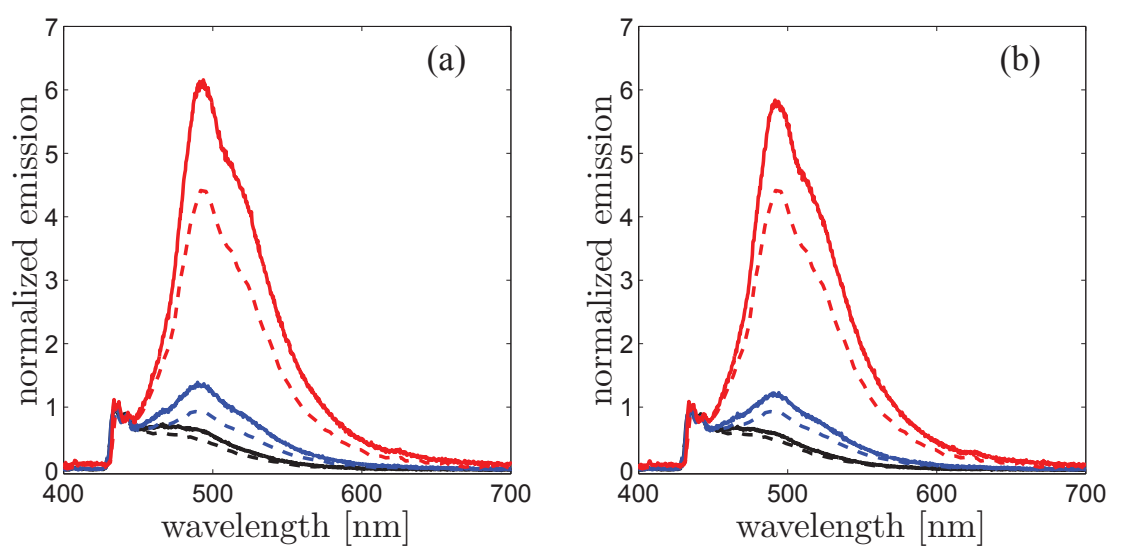

Figure 6: Normalized emission with respect to the monomer peak at $436 \mathrm{~nm}$ for three samples with dye concentration $0.1 \mathrm{wt} . \%$ (black), 0.5 wt.\% (blue), and $1.5 \mathrm{wt} . \%$ (red). (a) Emission of the virgin sample at the beginning of the test (solid line) and emission at the end of the loading phase for $\lambda \approx 2$ (dashed line). (b) Emission at the beginning of the unloading phase for $\lambda \approx 2$ (dashed line) and at the end of the cycle, when the sample is back to its undeformed configuration (solid line).

beginning of the test to the maximum inflation. Figure $7(\mathrm{a}-\mathrm{c})$ displays data for an inflation experiment with an equivalent strain rate of $0.013 \mathrm{~s}^{-1}$ and Figure $7(\mathrm{~d}-\mathrm{f})$ for an experiment with a smaller equivalent strain rate of $0.0057 \mathrm{~s}^{-1}$.

During inflation, the internal pressure shows an initial sharp increase associated with the initial expansion. 

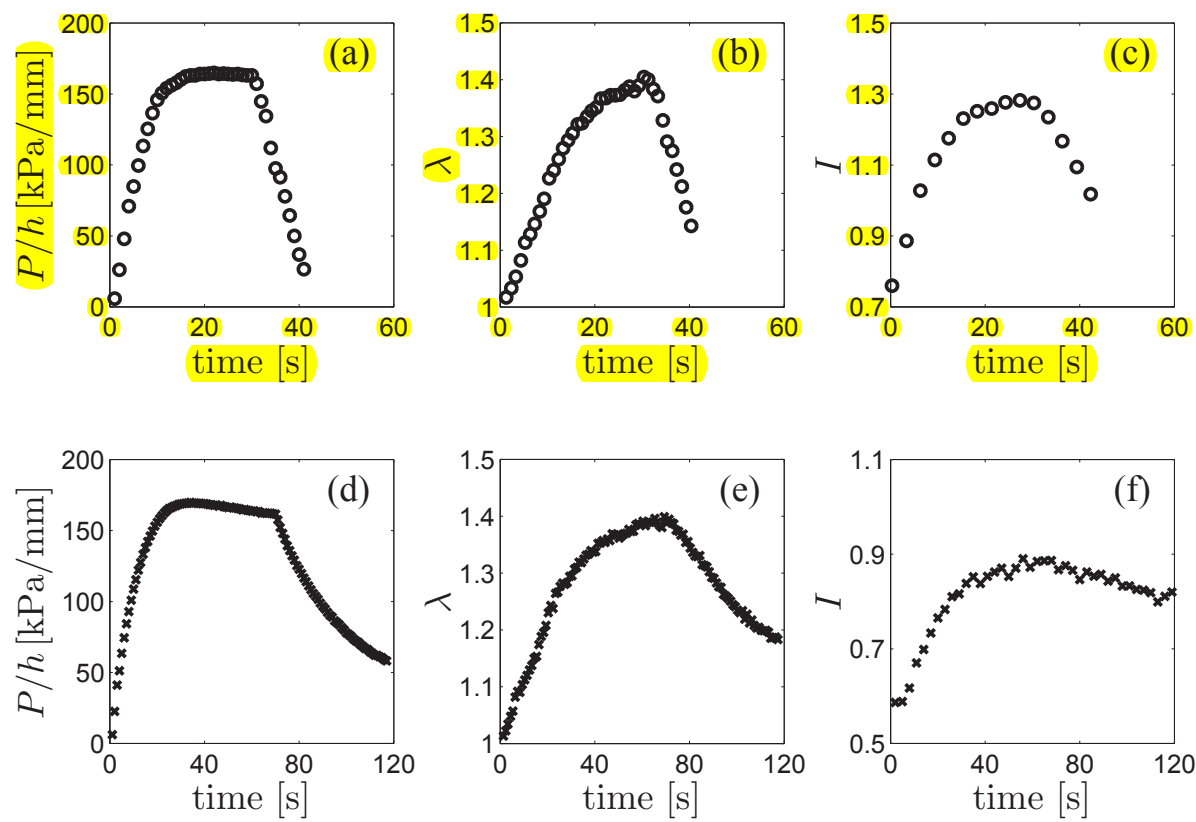

Figure 7: Internal pressure, stretch at the center of the membrane, and emission ratio during membrane inflation. (a-c) Inflation tests at an equivalent strain rate of $0.013 \mathrm{~s}^{-1}$ (circles); and (d-f) $0.0057 \mathrm{~s}^{-1}$ (crosses).

As the membrane progressively inflates, the rate of increase of the internal pressure is substantially reduced and the pressure reaches a maximum, while the membrane continues to expand (Rachik et al., 2001; Rivlin and Saunders, 1951). During deflation, an abrupt decrease of the internal pressure is observed, which results in a rapid reduction of the surface extension. Consistent with the uniaxial test, the emission ratio increases during inflation and decreases during deflation, although we note that the rate of decrease of the emission ratio during deflation is lower than the corresponding rate of increase during inflation, particularly for the slower strain rate, see Figures $7(\mathrm{~d}-\mathrm{f})$. As a result, the emission ratio is not entirely recovered after the deformation, whereby the final value of $I$ after deflation differs from the initial value prior to loading. We attribute the lower degree of reversibility of the mechanochromic response to a more severe damage of the polymer network in the inflation experiment as compared to the uniaxial test, which is also evidenced by the larger residual deformation of the membrane after stretching.

\subsection{The evolution of the microstructure regulates softening during cyclic loading}

Stress-strain curves generated from data in Figure 5 are presented in Figure 8(a). To identify the mechanical properties of different blends, we consider the response of the sample after relaxation. More specifically, we assume the material to reach a local equilibrium at the end of each relaxation period, and we define the equilibrium path as the locus of all the stress-strain values measured after relaxation (Cellini et al., 2014; Qi and Boyce, 2005). By identifying the material behavior from the equilibrium path, we are able to separate the time-independent stretching of the network from the time-dependent viscoelastic relaxation, which is not studied here.

In Figure 8(b), an experimental stress-strain curve is compared with the corresponding prediction from the constitutive model in equations (5), (6), and (8) for a $0.1 \mathrm{wt} . \%$ sample. We refer to the parameters utilized in Cellini et al. (2014) and Qi and Boyce (2005) for the analysis of large deformations of polyurethane elastomers. Specifically, the distance between two crosslinks is $N=6$, which determines the maximum chain extension in the network (Arruda and Boyce, 1993). The initial value of the volume fraction of soft phase is $v_{\mathrm{s} 0}=0.4$, the maximum volume fraction of soft phase is set to $v_{\mathrm{ss}}=0.85$, and the evolution rate is $A=1.4$. Following Bergstrom and Boyce (1999), we assume $f=4.7$ for the shape factor in equation (6). The value 

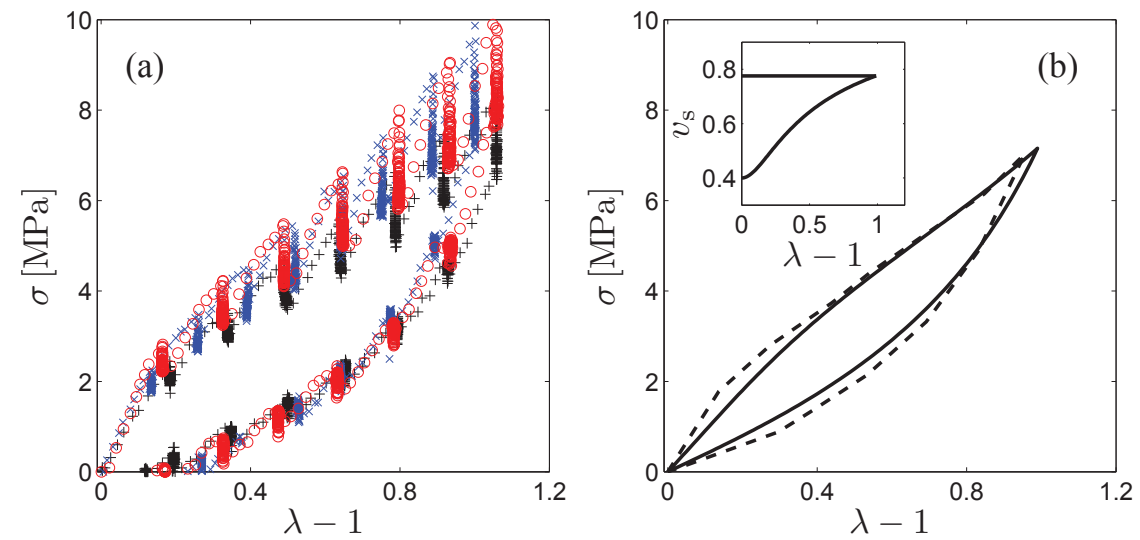

Figure 8: (a) True stress as a function of strain for three samples with dye concentrations $0.1 \mathrm{wt} . \%$ (black), 0.5 wt.\% (blue), and $1.5 \mathrm{wt. \%}$ (red). (b) The equilibrium path determined from experimental data ( $0.1 \mathrm{wt} \%$, dashed line) is compared with theoretical predictions (solid line). In the inset, the volume fraction of the soft phase as a function of the stretch is displayed.
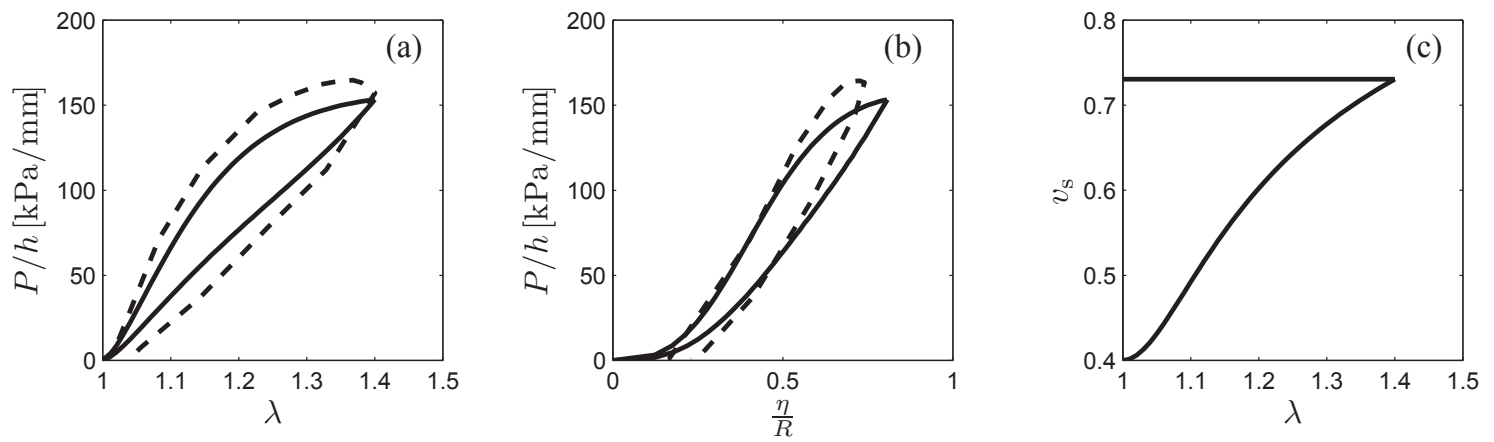

Figure 9: Internal pressure as a function of (a) the stretch and (b) the non-dimensional out-of-plane displacement at the center of the membrane. Dashed lines are experimental data and solid lines are predictions of the model in Appendix A. (c) Evolution of the volume fraction of the soft phase at the center of the membrane as a function of the stretch therein.

Table 1: Mean and standard deviation for mechanical properties measured during uniaxial loading of eight samples for each of the TPU-BBS blends considered $(0.1,0.5$, and $1.5 \mathrm{wt} . \%)$

\begin{tabular}{lllll}
\hline & $\sigma_{\max }(\mathrm{MPa})$ & $\lambda-1$ & $E(\mathrm{MPa})$ & $\mathrm{HR}$ \\
\hline $0.1 \mathrm{wt} \%$ & $7.63 \pm 0.87$ & $0.17 \pm 0.02$ & $13.19 \pm 1.91$ & $0.53 \pm 0.05$ \\
0.5 wt. $\%$ & $7.62 \pm 1.37$ & $0.14 \pm 0.04$ & $16.30 \pm 1.98$ & $0.46 \pm 0.06$ \\
1.5 wt.\% & $8.12 \pm 0.97$ & $0.17 \pm 0.02$ & $18.21 \pm 3.20$ & $0.50 \pm 0.05$ \\
\hline
\end{tabular}

of $\mu$ is identified by solving the following overdetermined system of equations in the least squares sense:

$$
\sum_{i=1}^{M}\left(\sigma_{i}-\sigma\left(\lambda_{i}, \mu\right)\right)=0,
$$

where $M$ is the number of points on the equilibrium path during the loading phase, $\sigma_{i}$ and $\lambda_{i}$ are experimental values of stress and stretch, and $\sigma$ is the theoretical prediction from equation (8). In the inset of Figure $8(\mathrm{~b})$, we display the variation of $v_{\mathrm{s}}$ as a function of $\lambda$ due to the progressive damage induced by large deformations of the polymer network. We identify $\mu=0.43 \pm 0.06 \mathrm{MPa}$ from aggregated data for the eight samples with concentration $0.1 \mathrm{wt} . \%, \mu=0.47 \pm 0.07 \mathrm{MPa}$ from samples with concentration $0.5 \mathrm{wt} . \%$, and $\mu=0.50 \pm 0.08 \mathrm{MPa}$ from samples with concentration $1.5 \mathrm{wt} . \%$.

To compare different experiments, we introduce the maximum stress on the equilibrium path $\sigma_{\max }$, the hysteresis ratio HR (the difference between the strain energy during the loading and unloading phases on 
the equilibrium path scaled by the strain energy during the loading), and the initial elastic modulus $E$ (the modulus of the material during the first loading step). In Table 1, we report the mean and standard deviation of mechanical parameters for the whole set of twenty-four virgin samples. We comment that the constitutive behavior of samples of different dye concentrations is highly comparable, in terms of maximum stress, hysteresis ratio, and residual plastic deformation, while experimental data show that samples with higher concentration have a slightly higher value of the initial elastic modulus. A similar increase of mechanical stiffness has been observed in polyurethane elastomer composites, when compatible fillers are incorporated in the polymer network (Pei et al., 2011; Wang and Pinnavaia, 1998).

Parameters identified from the uniaxial test are used in the analysis of biaxial loading during the membrane inflation test. To describe the mechanical behavior of the membrane, we use the same parameter values as in the uniaxial loading and we set $\mu=0.47 \mathrm{MPa}$, corresponding to $0.5 \mathrm{wt} \%$ samples. The constitutive behavior for biaxial deformations in equations (10) and (11) and equations (5) and (6) is used to predict the response of the material at the center of the membrane and the evolution of the microstructure during the inflation test. The full derivation and the solution of the equilibrium equations for axially symmetric inflation are detailed in Appendix A.

In Figure 9, we compare experimental data with theoretical predictions obtained through the solution scheme in Appendix A. In particular, Figure 9(a) and (b) display the pressure per unit thickness as a function of the stretch $\lambda$ at the center of the membrane and the non-dimensional out-of-plane displacement $\eta / R$ at the center of the membrane. In addition, Figure $9(\mathrm{c})$ displays the evolution of the volume fraction of the soft phase during the deformation cycle at the center of the membrane. The evolution of the soft phase is comparable to that observed for uniaxial loading, even if the stretch levels are considerably different. Notably, the conversion of the hard phase in the soft phase depends on the membrane extension $\Lambda_{\text {chain }}^{\text {max }}$ through the first invariant $J_{1}$, therefore biaxial deformations result in more severe damage of the polymer structure as compared to uniaxial extension for equivalent strain values.

\subsection{Dye concentration and network stretching influence the optical response}

Figure 10(a) and (b) shows the variation of the monomer to excimer emission ratio as a function of the stretch $\lambda$ for dye concentrations $0.1,0.5$, and $1.5 \mathrm{wt} . \%$ for the loading and unloading cycles, respectively. We calibrate the constitutive model in equation (9) by identifying the values of the parameters $I_{0}$ and $\alpha$ during the loading cycle for eight samples for each concentration. We present aggregated data obtained for the three different concentrations in Table 2. Data presented in Figure 10(a) and (b) show that the mechanochromic response of TPU-BBS blends is largely reversible and controlled by dye concentration in the polymer.

The initial dye concentration has an effect on the initial emission ratio and on the relative shift of the emission from the excimer to the monomer bands. Data presented in Table 1 indicate that the initial emission ratio decreases from $1.32 \pm 0.14$ to $0.13 \pm 0.03$ as the dye concentration increases from 0.1 to $1.5 \mathrm{wt} . \%$. This result is expected as the increased concentration of dye in the polymer is likely to induce formation of aggregates and consequently shift the emission towards the excimer band. Furthermore, substantial variations of the parameter $\alpha$ are observed for different concentrations of dye in the polymer, with the largest value of $\alpha=0.62 \pm 0.25$ obtained for $0.5 \mathrm{wt} . \%$. We suggest that the increase of $\alpha$ between 0.1 and 0.5 wt. $\%$ could be attributed to the increased amount of dye aggregates, which result in a more pronounced mechanochromic response at $0.5 \mathrm{wt} . \%$. The decrease of $\alpha$ at $1.5 \mathrm{wt} . \%$ may be related to the progressive quenching of monomer fluorescence by dye aggregates, which results in a consistent decrease of the values of the emission ratio $I$ and, in turn, of the values of $\alpha$.

In Figure 10(c), we compare emission ratios measured during the membrane inflation in tests shown in Figure $7(\mathrm{c})$ and (e) with predictions of the constitutive behavior in equation (7), using parameters identified from the uniaxial tests. The values of the parameters $\alpha$ and $I_{0}$ correspond to samples with dye concentration 0.5 wt. $\%$, as reported in Table 2. To compute the integral in equation (12), we assume that the stretch on the top surface of the membrane, where we acquire the fluorescence signal, equals the stretch at the midsurface, consistent with the derivation in Appendix A. The shaded area reported in Figure 10(c) is the error band associated with the determination of the parameter $\alpha$ and $I_{0}$ in Table 2. Results for the two tests in Figure 10(c), executed on two different membrane samples, are both within one standard deviation 

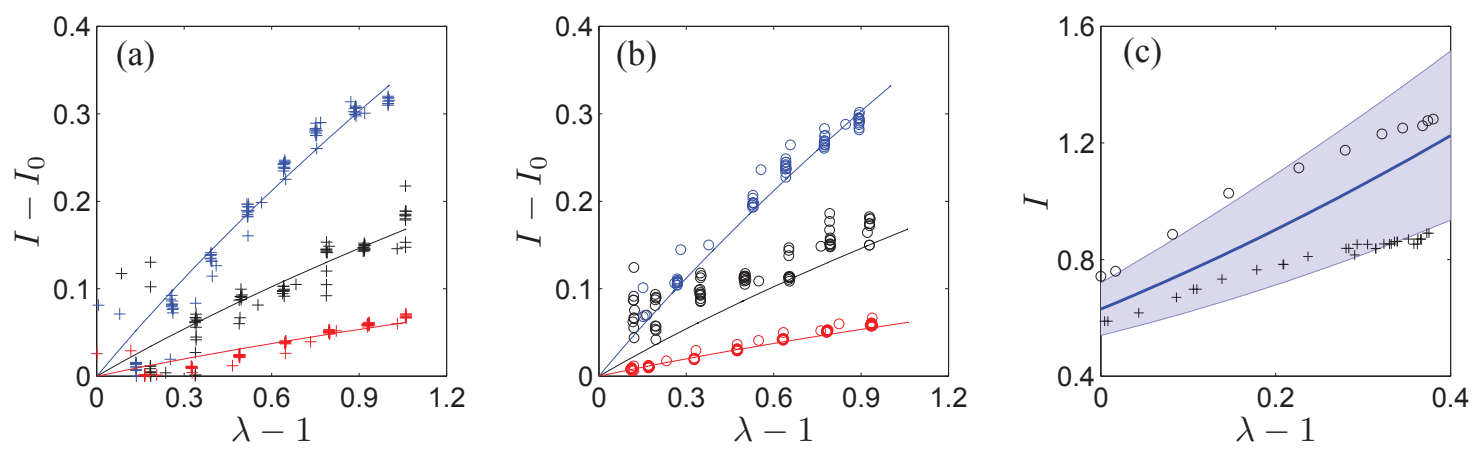

Figure 10: (a) Emission ratio as a function of dye concentration in the polymer during the loading cycle. Black markers are for $0.1 \mathrm{wt} . \%$, blue markers for $0.5 \mathrm{wt} . \%$, and red markers for $1.5 \mathrm{wt} . \%$ concentrations, respectively. Lines represent fitting with equation (9). (b) Emission ratio as a function of dye concentration in the polymer during the unloading cycle. Black markers are for $0.1 \mathrm{wt} . \%$, blue markers for $0.5 \mathrm{wt} . \%$, and red markers for $1.5 \mathrm{wt} . \%$ concentrations, respectively. Lines represent fitting with equation (9) using parameters identified during the loading cycle in subfigure (a). (c) Emission ratio measured for membrane inflation at $0.013 \mathrm{~s}^{-1}$ (circles) and $0.0057 \mathrm{~s}^{-1}$ (crosses). Markers are experimental findings and the solid line is the theoretical prediction from equation (12) with parameters given by the average values in Table 2 for $0.5 \%$ dye concentration, $\lambda$ is the stretch at the center of the membrane. The shaded region represents the predictions of equation (12) by varying $\alpha$ and $I_{0}$ from their average values within one standard deviation in Table 2.

Table 2: Mean and standard deviation for optical properties measured during uniaxial loading of eight samples for each of the TPU-BBS blends $(0.1,0.5$, and $1.5 \mathrm{wt} . \%)$.

\begin{tabular}{lll}
\hline & $I_{0}$ & $\alpha$ \\
\hline 0.1 wt. $\%$ & $1.32 \pm 0.14$ & $0.40 \pm 0.15$ \\
0.5 wt. $\%$ & $0.63 \pm 0.09$ & $0.62 \pm 0.25$ \\
1.5 wt. $\%$ & $0.13 \pm 0.03$ & $0.16 \pm 0.02$ \\
\hline
\end{tabular}

from the predicted behavior, supporting the accuracy of the proposed constitutive hypothesis. However, we cannot dismiss the possibility that time-dependent phenomena could play a role on mechanochromic response, causing a dependence of the emission ratio on the time history of the deformation, different from equation (7).

\subsection{The extent of the optical response is not reduced over successive loading cycles}

Experimental data for the nominal stress, stretch, and emission ratio acquired during cyclic uniaxial testing of a TPU-BBS sample with $0.5 \mathrm{wt} . \%$ dye concentration are displayed in Figure 11. Experimental data show that deformation of the film after the first cycle is almost completely recovered as the applied load is removed. We also observe a partial recovery of the initial emission ratio after the first cycle. Subsequent cycles show more consistent recovery of deformation and emission ratio, with the respective values at the end of a given cycle being nearly identical to that at the start of the cycle.

Stress-strain curves from data recorded during the cyclic tests are displayed in Figure 12(a-c). Figure 12(a) refers to the first cycle, wherein the dashed line represents the equilibrium path inferred from the values of the stress and strain obtained after relaxation for $60 \mathrm{~s}$. Figure 12(b) and (c) pertain to the second and third loading cycles, respectively. We use condition (4) to determine the stress-strain curves for successive loading cycles, along with equations (5), (6), and (8). Based on equation (4), the volume fraction of the soft phase $v_{\mathrm{s}}$ increases only when $\Lambda_{\text {chain }}>\Lambda_{\text {chain }}^{\max }$, that is, when the stretch of the polymer network is larger than the maximum stretch experienced during the loading history of the sample. Thus, the evolution of the microstructure is locked during loading on successive cycles until the network stretch overcomes the value $\Lambda_{\text {chain }}^{\max }$ obtained during the previous cycles. Therefore, the loading path for the second cycle corresponds to the unloading path of the first cycle, and so on.

Figure 13(a) and (b) display theoretical stress-strain curves for three successive cycles together with the value of $v_{\mathrm{s}}$ obtained from the evolution law in equation (5) subject to the condition in equation (4). The 

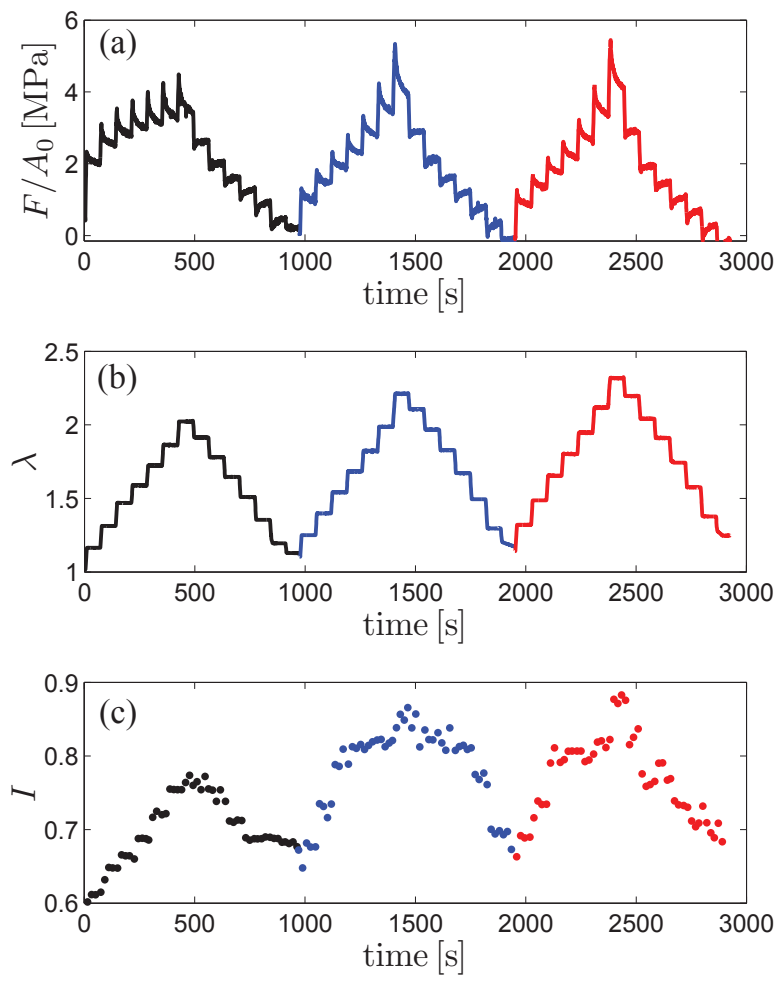

Figure 11: (a) Nominal stress, (b) stretch, and (c) emission ratio as a function of time for a cyclic test performed on a 0.5 wt.\% sample. The black curves corresponds to the first loading cycle, while blue and red curves correspond to the second and third cycles, respectively.

model predicts a consistent softening of the equilibrium path as well as a reduction of the hysteresis ratio for the second and third loading cycle. Equilibrium paths identified from experiments and results from the constitutive model are compared in Figure $12(\mathrm{~d}-\mathrm{e})$. The parameter $\mu=0.43 \mathrm{MPa}$ is identified on the first cycle and theoretical stress-strain curves for successive cycles are determined by retaining this value. Moreover, we assume the value of the shape factor $f=4.7$ to remain unchanged over repeated loading cycles.

In Figure 14(a-f), we present the emission ratio recorded over successive loading-unloading cycles as a function of the sample stretch. To interpret the optical response of the material, we adopt the constitutive behavior in equation (9). In particular, we use the values of the emission ratio from the first loading cycle in Figure 14(a) to identify the parameter $\alpha=0.44$. The good agreement between the proposed constitutive relationship, calibrated on the first loading cycle, for successive loading cycles offer indirect evidence for the reversibility of the mechanochromic response. The parameter $I_{0}$ is determined independently for each cycle. We find $I_{0}=0.59$ for the first cycle, $I_{0}=0.65$ for the second cycle, and $I_{0}=0.66$ for the third cycle. The increase of the emission ratio at the end of the first cycle can be related to the permanent conversion of a small part of the aggregates to the monomeric phase.

\section{Summary and Conclusions}

In this study, we investigated reversible mechanochromism of polyurethane elastomers blended with bis(benzoxazolyl)stilbene dye. Material response to uniaxial loading was studied in stress relaxation experiments, while biaxial loading was investigated in a set of membrane inflation tests. Experiments were designed to simultaneously measure the mechanical and optical responses of the material and dissect the relationship between material deformation and variations of the monomer to excimer emission ratio. Mechanochromism 

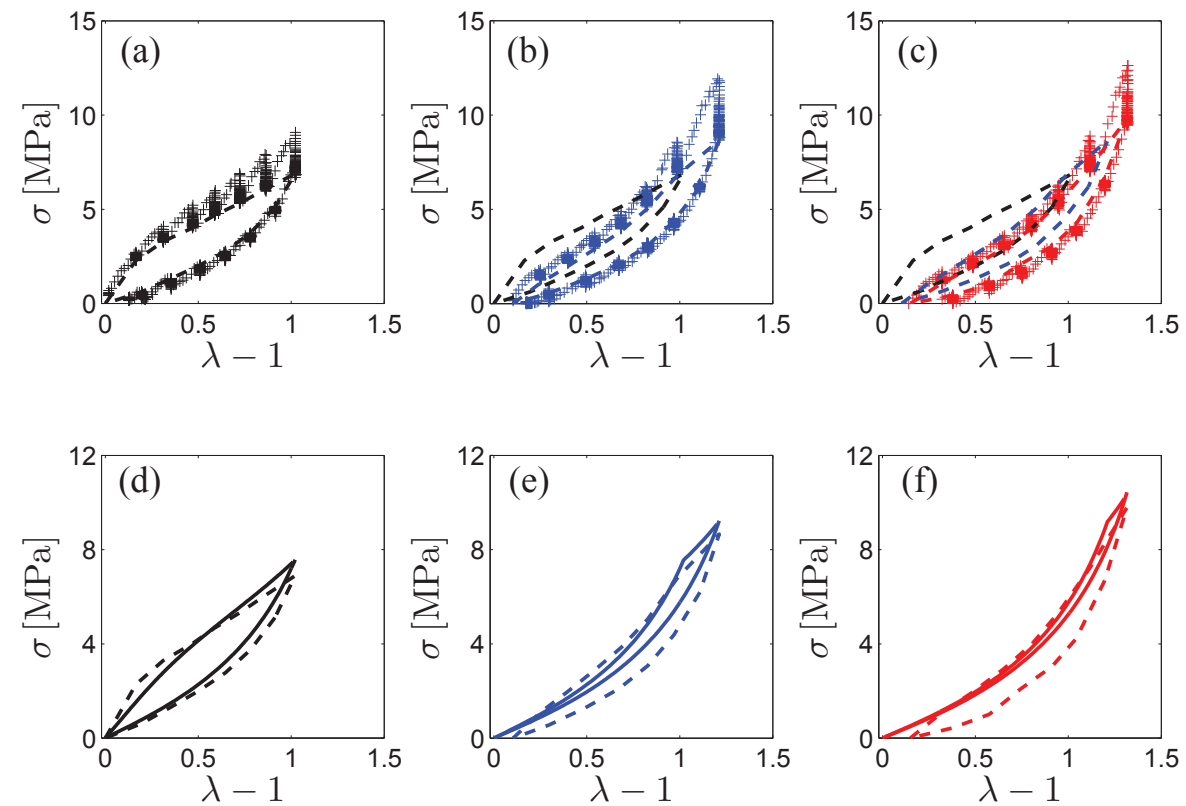

Figure 12: True stress as a function of strain during the loading-unloading cycles. Markers are experimental data for the (a) first cycle; (b) second cycle; and (c) third cycle. Dashed lines represent equilibrium paths determined after relaxation of stresses for $60 \mathrm{~s}$ during each cycle. (d-f) Theoretical predictions from the constitutive model (solid lines) are compared with equilibrium paths identified for the three cycles (dashed lines) in $(\mathrm{a}-\mathrm{c})$.
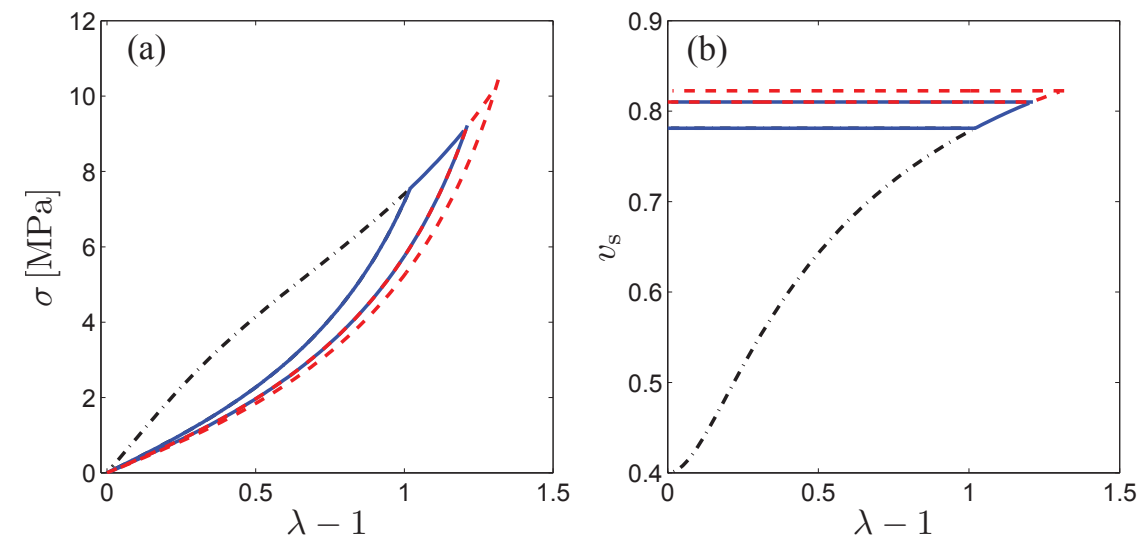

Figure 13: (a) Theoretical stress-strain curves for a cyclic experiment. (b) Evolution of the volume fraction of the soft phase. Black dash-dotted lines are for the first cycle, blue solid lines for the second cycle, and red dashed lines for the third cycle. Note that lines between two consecutive cycles partly overlap.

of TPU-BBS blends was observed during large deformations under both uniaxial and biaxial loading. Variations of the emission ratio were attributed to reorganization of dye aggregates within the elastomeric network, with a relative increase of the emission in the monomer band during loading and an increase of emission in the excimer band during unloading.

Experimental data revealed a dependence of the optical response on the mechanical deformation of the polymer. In particular, experimental data for uniaxial and biaxial loading suggest a linear dependence of the emission ratio on the overall surface stretch of the film, measured as the ratio between the deformed and underformed surface areas. However, experimental results indicated a small disparity between the initial and final values of the emission ratio in the first loading cycle, which is likely due to a permanent increase 

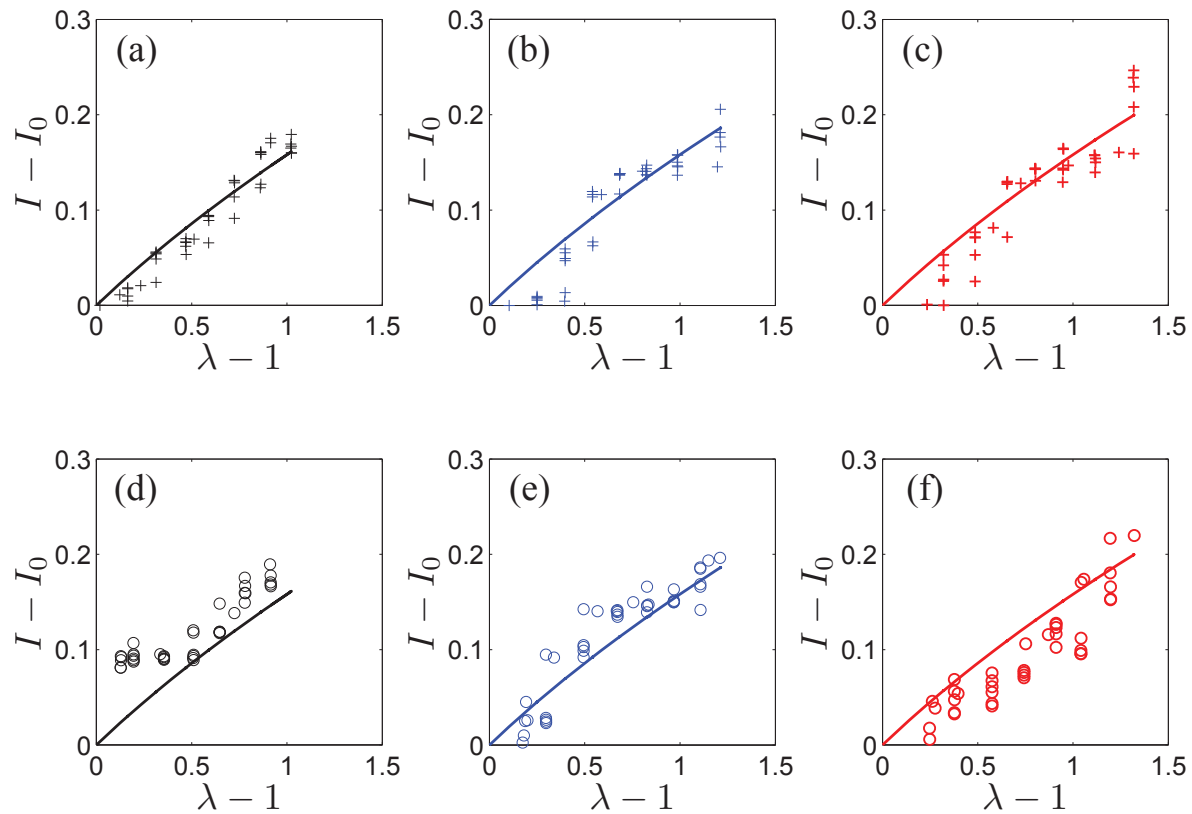

Figure 14: Emission ratio as a function of the stretch for a cyclic experiment. Markers are experimental data for the loading phase for (a) the first cycle; (b) the second cycle; and (c) the third cycle; and the unloading phase for (d) the first cycle; (e) the second cycle; and (f) the third cycle. The solid line is the prediction from the constitutive model in equation (9) for $\alpha=0.44$ identified on the first loading cycle. Values of $I_{0}$ are determined independently for each cycle: $I_{0}=0.59$ for the first cycle; $I_{0}=0.65$ for the second cycle; and $I_{0}=0.66$ for the third cycle.

of the monomeric phase during stress recovery. Similar hysteretic mechanochromic responses have been found in other polymer-dye blends (Kunzelman et al., 2006; Pucci et al., 2005, 2011). Repeated mechanical loading did not abate the mechanochromic response associated with the stretch of the network and positively contributed to its reversibility. This suggests that after an initial permanent increase of the monomeric phase in the first loading cycle, subsequent cycles did not produce further irreversible modifications.

The experimentally observed permanent modifications in the optomechanical response of the material after large deformation was modeled by assuming progressive damage of the biphasic structure of the polymer network; that is, a permanent increase in the volume fraction of the soft phase of the polymer. Using this assumption, we developed a constitutive model that closely describes the observed stress-strain behavior of the samples during loading-unloading cycles for uniaxial and biaxial deformations. The evolution of the microstructure during large deformations of the polymer network captures the progressive degradation of mechanical properties and softening of the equilibrium path. This non-reversible evolution of the elastomer structure also explains the disparity in the emission ratio observed in the first loading cycle of the experiments.

The optical response of the TPU-BBS blends was found to be a function of the concentration of dye in the polymer. Calibrating the constitutive relationship for the fluorescence emission on experimental observations showed that optical response of the polymer during uniaxial loading depended on the initial emission ratio, which was a decreasing function of the dye concentration. Such a result was initially expected, as higher concentrations of dye in the polymer are known to ease the formation of dye aggregates (Bao et al., 2013; Pucci et al., 2005), leading to an increase of emission in the excimer band, corresponding to a reduction of the emission ratio. The initial dye concentration also regulated the relative variation of monomer and excimer emissions during stretching, through a constitutive parameter measuring the rate of variation of the emission ratio with respect to the change in surface area. In particular, TPU-BBS blends with 0.5 wt.\% presented a higher relative variation of the emission ratio during stretching in comparison to blends with dye concentrations of $0.1 \mathrm{wt} . \%$ and $1.5 \mathrm{wt} . \%$. This result is relevant for the design of mechanochromic sensors, as initial dye concentration can potentially be used to regulate the sensitivity of the device. 
The optomechanical behavior of mechanochromic elastomer described in this manuscript will aid in the design of non-contact optical strain sensors and force transducers, with applications in mechanics, biomechanics, and life sciences. In particular, we envision the application of mechanochromic sensors in fluid mechanics, for minimally-invasive measurement of pertinent field variables, including pressure, temperature, salinity, and $\mathrm{pH}$, to name a few.

\section{Aknowledgements}

This research was supported by the National Science Foundation through Grant No. CBET-1332204. The authors also acknowledge support from the Office of Naval Research through Grant No. N00014-10-10988, which has allowed the acquisition of equipment used in this study. The authors want to thank Dr. Avi Ulman and the Institute for Engineered Interfaces (IEI) at the New York University Tandon School of Engineering for granting access to the laboratories and Steven Osma for his help in the experiments.

\section{Appendix A. Axially symmetric membrane inflation}

Here, we present a derivation and solution of the governing equations for axially symmetric inflation of an initially flat membrane of radius $R$ and thickness $h$ composed of a material whose constitutive behavior is described by equations (2), (5), and (6). The geometry of the membrane is defined through a cylindrical set of coordinates $(r, \theta, z)$, where $r$ and $z$ run along the membrane radius and thickness in the undeformed configuration, and $\theta$ is the azimuthal angle. We set the origin of the coordinate system at the center of the membrane. Upon axially symmetric inflation, for each angle $\theta$, a point $Q$ of coordinates $(r, 0)$ on the membrane mid-surface at a radius $r$ in the reference configuration will occupy the coordinates $(\rho, \eta)$, where $\rho$ is along the undeformed membrane and $\eta$ is orthogonal to it, see the schematic in Figure A.15. The origin of such a new coordinate system coincides with the origin of $(r, \theta, z)$. We define the stretches of the membrane mid-surface in the meridian (subscript 1) and circumferential (subscript 2) directions as (Adkins and Rivlin, 1952; Goulbourne et al., 2005; Yang and Feng, 1970)

$$
\begin{gathered}
\lambda_{1}=\sqrt{1+\left(\frac{\mathrm{d} \eta}{\mathrm{d} \rho}\right)^{2}} \frac{\mathrm{d} \rho}{\mathrm{d} r}, \\
\lambda_{2}=\frac{\rho}{r},
\end{gathered}
$$

and we determine the stretch component along the thickness $\lambda_{3}$ by assuming the membrane to be incompressible, so that $\lambda_{3}=\frac{1}{\lambda_{1} \lambda_{2}}$.

Following Love (1906) and Adkins and Rivlin (1952), the equilibrium equations are derived from the equilibrium of forces acting on a surface element of the membrane in the deformed configuration. For a thin membrane undergoing axially symmetric deformations, flexural and shear stresses are negligible. Thus, forces acting on the membrane are determined from the equilibria along the meridian and circumferential directions as (Adkins and Rivlin, 1952; Goulbourne et al., 2005; Yang and Feng, 1970)

$$
\begin{gathered}
\frac{\mathrm{d} T_{1}}{\mathrm{~d} \rho}=\frac{T_{2}-T_{1}}{\rho}, \\
k_{1} T_{1}+k_{2} T_{2}=P,
\end{gathered}
$$

where $T_{1}$ and $T_{2}$ are the resultant forces along the meridian and circumferential directions, $k_{1}$ and $k_{2}$ are the mid-surface curvatures, and $P$ is the internal pressure. The tensions $T_{1}$ and $T_{2}$ are obtained under the assumption that stresses are constant within the thickness of the membrane (Adkins and Rivlin, 1952; Goulbourne et al., 2005; Yang and Feng, 1970), so that from equations (10) and (11) we find

$$
T_{1}=h \lambda_{3} \sigma_{1}=h \beta\left(\frac{\lambda_{1}}{\lambda_{2}}-\frac{1}{\lambda_{1}^{3} \lambda_{2}^{3}}\right),
$$




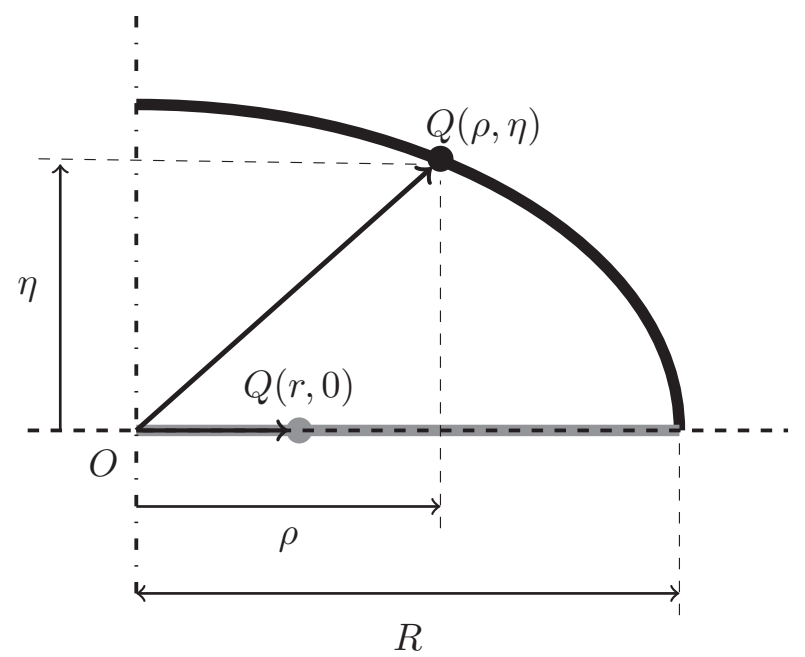

Figure A.15: Schematic of the membrane in the reference configuration (gray line) and during axial symmetric inflation (black line). The dash-dotted line is an axis of rotational symmetry.

$$
T_{2}=h \lambda_{3} \sigma_{2}=h \beta\left(\frac{\lambda_{2}}{\lambda_{1}}-\frac{1}{\lambda_{1}^{3} \lambda_{2}^{3}}\right),
$$

where $\beta\left(\lambda_{1}, \lambda_{2}\right)=\frac{v_{\mathrm{s}} X \mu}{3} \frac{\sqrt{N}}{\Lambda_{\text {chain }}} \mathrm{L}^{-1}\left(\frac{\Lambda_{\text {chain }}}{\sqrt{N}}\right)$. The solution of the compatibility equations (Adkins and Rivlin, 1952) yields the curvatures $k_{1}$ and $k_{2}$ as

$$
\begin{gathered}
k_{1}=\frac{\frac{\mathrm{d} \rho}{\mathrm{d} r} \frac{\mathrm{d} \lambda_{1}}{\mathrm{~d} r}-\lambda_{1} \frac{\mathrm{d}^{2} \rho}{\mathrm{d} r^{2}}}{\lambda_{1}^{2} \sqrt{\lambda_{1}^{2}-\left(\frac{\mathrm{d} \rho}{\mathrm{d} r}\right)^{2}}}, \\
k_{2}=\frac{\sqrt{\lambda_{1}^{2}-\left(\frac{\mathrm{d} \rho}{\mathrm{d} r}\right)^{2}}}{r \lambda_{1} \lambda_{2}} .
\end{gathered}
$$

By substituting equations (A.5), (A.6), (A.7), and (A.8) into equations (A.3) and (A.4), we find the following system of differential equations for $\lambda_{1}, \lambda_{2}$, and $\rho$ :

$$
\begin{aligned}
& \frac{\mathrm{d} \lambda_{1}}{\mathrm{~d} r}=\frac{1}{\left(\frac{1}{\lambda_{2}}+\frac{3}{\lambda_{1}^{4} \lambda_{2}^{3}}\right)+\frac{1}{\beta} \frac{\mathrm{d} \beta}{\mathrm{d} \lambda_{1}}\left(\frac{\lambda_{1}}{\lambda_{2}}-\frac{1}{\lambda_{1}^{3} \lambda_{2}^{3}}\right)} \times \\
& \times\left(\frac{\mathrm{d} \lambda_{2}}{\mathrm{~d} r}\left(\frac{\lambda_{1}}{\lambda_{2}^{2}}-\frac{3}{\lambda_{1}^{3} \lambda_{2}^{4}}\right)-\frac{\mathrm{d} \lambda_{2}}{\mathrm{~d} r} \frac{1}{\beta} \frac{\mathrm{d} \beta}{\mathrm{d} \lambda_{2}}\left(\frac{\lambda_{1}}{\lambda_{2}}-\frac{1}{\lambda_{1}^{3} \lambda_{2}^{3}}\right)-\frac{\mathrm{d} \rho}{\mathrm{d} r} \frac{1}{\lambda_{2} r}\left(\frac{\lambda_{1}}{\lambda_{2}}-\frac{\lambda_{2}}{\lambda_{1}}\right)\right) \\
& \frac{\mathrm{d} \lambda_{2}}{\mathrm{~d} r}=\frac{\frac{\mathrm{d} \rho}{\mathrm{d} r}-\lambda_{2}}{r} \\
& \frac{\mathrm{d}^{2} \rho}{\mathrm{d} r^{2}}=\frac{1}{\lambda_{1}} \frac{\mathrm{d} \rho}{\mathrm{d} r} \frac{\mathrm{d} \lambda_{1}}{\mathrm{~d} r}+\frac{\lambda_{1} \sqrt{\lambda_{1}^{2}-\left(\frac{\mathrm{d} \rho}{\mathrm{d} r}\right)^{2}}}{\frac{\lambda_{1}}{\lambda_{2}}-\frac{1}{\lambda_{1}^{3} \lambda_{2}^{3}}} \frac{1}{r}\left(\frac{\sqrt{\lambda_{1}^{2}-\left(\frac{\mathrm{d} \rho}{\mathrm{d} r}\right)^{2}}}{\lambda_{1} \lambda_{2}}\left(\frac{\lambda_{2}}{\lambda_{1}}-\frac{1}{\lambda_{1}^{3} \lambda_{2}^{3}}\right)-\frac{P r}{\beta h}\right),
\end{aligned}
$$

where $\frac{d \beta}{d \lambda_{1}}$ and $\frac{d \beta}{d \lambda_{2}}$ are determined by approximating the inverse Langevin function following Jedynak (2014) 

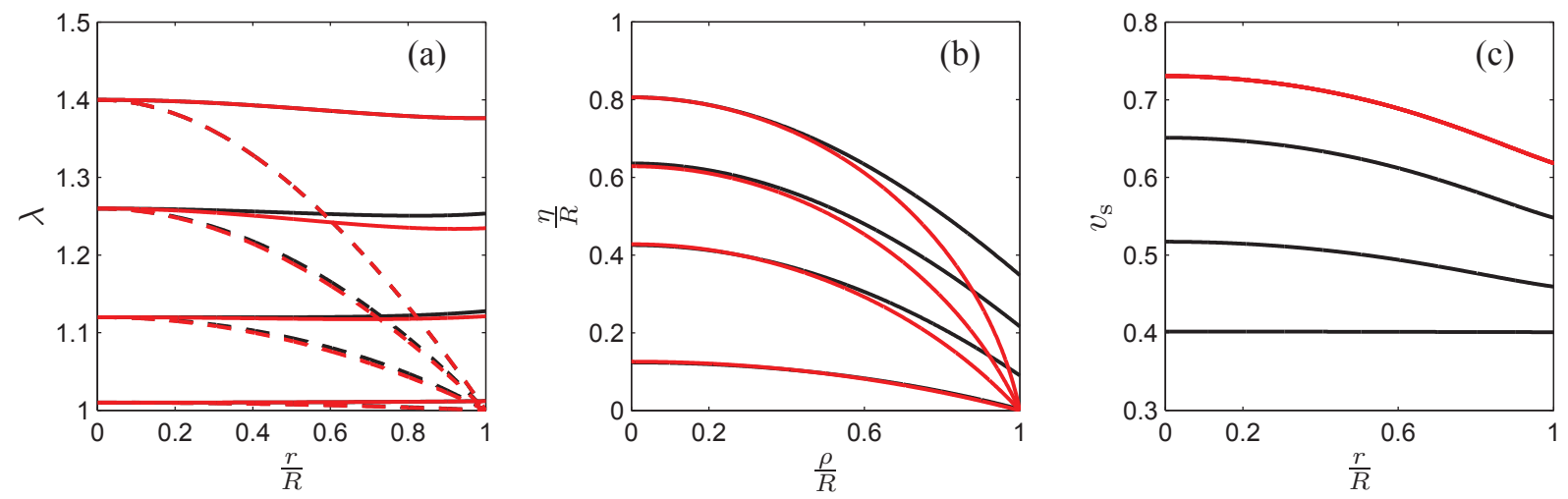

Figure A.16: (a) Meridian (solid) and circumferential (dashed) stretches as a function of $r$ for increasing stretch at the center of the membrane. (b) Membrane profile for increasing stretch at the center of the membrane. (c) Volume fraction of soft segments for increasing stretch at the center of the membrane. Black lines correspond to virgin membranes, while red lines correspond to predeformed membranes with $\lambda_{1}=\lambda_{2}=1.4$ at the center of the membrane.

as $\mathrm{L}^{-1}(x) \approx x \frac{3-x^{2}}{1-x^{2}}$, so that

$$
\frac{\mathrm{d} \beta}{\mathrm{d} \lambda_{1}}=-\frac{v_{s} X^{2} \mu}{9 N} \frac{2 \lambda_{1}-\frac{2}{\lambda_{1}^{3} \lambda_{2}^{2}}}{1-\left(\frac{\Lambda_{\text {chain }}}{\sqrt{N}}\right)^{2}}+\frac{v_{s} X^{2} \mu}{9 N} \frac{\left(2 \lambda_{1}-\frac{2}{\lambda_{1}^{3} \lambda_{2}^{2}}\right)\left(3-\left(\frac{\Lambda_{\text {chain }}}{\sqrt{N}}\right)^{2}\right)}{\left(1-\left(\frac{\Lambda_{\text {chain }}}{\sqrt{N}}\right)^{2}\right)^{2}},
$$

and

$$
\frac{\mathrm{d} \beta}{\mathrm{d} \lambda_{2}}=-\frac{v_{s} X^{2} \mu}{9 N} \frac{2 \lambda_{2}-\frac{2}{\lambda_{1}^{2} \lambda_{2}^{3}}}{1-\left(\frac{\Lambda_{\text {chain }}}{\sqrt{N}}\right)^{2}}+\frac{v_{s} X^{2} \mu}{9 N} \frac{\left(2 \lambda_{2}-\frac{2}{\lambda_{1}^{2} \lambda_{2}^{3}}\right)\left(3-\left(\frac{\Lambda_{\text {chain }}}{\sqrt{N}}\right)^{2}\right)}{\left(1-\left(\frac{\Lambda_{\text {chain }}}{\sqrt{N}}\right)^{2}\right)^{2}}
$$

Boundary conditions for the equations (A.9), (A.10), (A.11) are assigned at the center of the membrane and at the external clamp. Given the symmetry of the problem, we assume that stretches in the circumferential and meridian directions are equal at the center of the membrane, that is, $\lambda_{1}=\lambda_{2}$ at $r=0$. From the clamping condition, we have that $\lambda_{2}=1$ at $r=R$.

Equations (A.9), (A.10), and (A.11) are controlled by the parameters $v_{s}$ and $X$, which, in turn, depend on the stretches $\lambda_{1}$ and $\lambda_{2}$ through equations (5) and (6). The solution of equations (5) and (6) is computed as a function of the chain stretch $\Lambda_{\text {chain }}$. Thus, values of $v_{s}$ and $X$ as functions of $\Lambda_{\text {chain }}$ are determined in a tabular form for given values of $N$ and $\mu$. Such values for $v_{\mathrm{s}}$ and $X$ are, in turn, used to solve equations (A.9), (A.10), and (A.11) and determine a tabular form for $\beta\left(\lambda_{1}, \lambda_{2}\right), \frac{\mathrm{d} \beta}{\mathrm{d} \lambda_{1}}\left(\lambda_{1}, \lambda_{2}\right)$, and $\frac{\mathrm{d} \beta}{\mathrm{d} \lambda_{2}}\left(\lambda_{1}, \lambda_{2}\right)$.

The solution of equations (A.9), (A.10), and (A.11) is obtained by first reducing the order of the problem through the auxiliary variable $\omega=\frac{\mathrm{d} \rho}{\mathrm{d} r}$ and then using the iterative procedure presented in Yang and Feng (1970). At the beginning of the iterative procedure, we assign the value of the stretch at the center of the membrane and an initial guess of the the internal pressure. Thus, we integrate equations (A.9), (A.10), and (A.11) from $r=0$ using a $4^{\text {th }}$ order Runge-Kutta scheme, where the values of $v_{\text {s }}$ and $X$ are computed at each integration step by fitting the tabulated data. Numerical integration is continued until the condition at the external boundary $\lambda_{2}=1$ is satisfied. In general, this condition is not satisfied at $r=R$ for the initial guess of the internal pressure. Following Yang and Feng (1970), we introduce the scaling factor $\gamma=\frac{R}{r_{f}}$ where $r_{\mathrm{f}}$ is the radius at which the solution satisfies the whole set of boundary conditions. We compute the new value of the internal pressure at the successive step as $P_{j+1}=\frac{P_{j}}{\gamma}$, where $j$ is an index indicating the iteration. The procedure is iterated until $r_{\mathrm{f}}=R$. 
In Figure A.16, we show model predictions for $N=6, \mu=0.47 \mathrm{MPa}$, and $v_{\mathrm{s} 0}=0.4$ at $t=0, v_{\mathrm{ss}}=0.85$, and $A=1.4$. We present results for two different conditions, whereby we consider an initially virgin membrane as well as a membrane that is deformed after a first deformation cycle with a maximum stretch of $\lambda_{1}=\lambda_{2}=1.4$ at the center of the membrane. For the latter case, based on condition (4), $v_{\mathrm{s}}$ and $X$ maintain the values attained for $\lambda_{1}=\lambda_{2}=1.4$ until $\Lambda_{\text {chain }}>\Lambda_{\text {chain }}^{\max }$, that is, $\lambda_{1}, \lambda_{2}>1.4$.

Values of the stretches $\lambda_{1}$ and $\lambda_{2}$, the deformed shape of the membrane mid-surface, and the volume fraction $v_{\mathrm{s}}$ as functions of the radial coordinate $r$ are displayed in Figure A.15(a-c) for increasing values of the stretch at $r=0$. The value of $v_{\mathrm{s}}$ increases for the virgin material by increasing the stretch of the membrane, while it maintains a constant value for the predeformed membrane. Moreover, we note that $v_{\mathrm{s}}$ has a maximum at $r=0$ and a minimum at the clamp, where the value of the chain stretch $\Lambda_{\text {chain }}$ is determined by the sole meridian stretch $\lambda_{1}$. We further notice that the shape of the membrane is affected by the initial damage, whereby a change in the meridian stretch is noted in the vicinity of the clamp. We comment that for the case of the initially deformed membrane, the same value of the stretch at $r=0$ is obtained for a lower value of the pressure than for the virgin membrane due to the softening of the equilibrium path, see also Figure 9.

\section{References}

Adkins, J.E., Rivlin, R.S., 1952. Large elastic deformation of isotropic materials. IX. The deformation of thin shells. Philosophical Transactions of the Royal Society of London A 244, 505-531.

Arruda, E., Boyce, M.C., 1993. A three-dimensional constitutive model for the large stretch behavior of rubber elastic materials. Journal of the Mechanics and Physics of Solids 41, 389-412.

Bao, S., Li, J., Lee, K.I., Shao, S., Hao, J., Fei, B., Xin, J.H., 2013. Reversible mechanochromism of a luminescent elastomer. Applied Materials and Interfaces 5, 4625-4631.

Beiermann, B.A., Kramer, S.L.B., May, P.A., Moore, J.S., White, S.R., Sottos, N.R., 2014. The effect of polymer chain alignment and relaxation on force-induced chemical reactions in an elastomer. Advanced Functional Materials 24, 15291537.

Bergstrom, J.S., Boyce, M.C., 1999. Mechanical behavior of particle filled elastomers. Rubber Chemistry and Technology 72 , 633-656.

Bergstrom, J.S., Boyce, M.C., 2000. Large strain time-dependent behavior of filled elastomers. Mechanics of Materials 32, 627 -644 .

Castaneda, P.P., 1989. The overall constitutive behaviour of nonlinearly elastic composites. Proceedings of the Royal Society of London A: Mathematical, Physical and Engineering Sciences 422, 147-171.

Cellini, F., Khapli, S., Peterson, S.D., Porfiri, M., 2014. Mechanochromic polyurethane strain sensor. Applied Physics Letters 105,061907

Chan, E.P., Walish, J.J., Urbas, A.M., Thomas, E.L., 2013. Mechanochromic photonic gels. Advanced Materials 25, 3934-3947.

Chen, Y., Sijbesma, R.P., 2014. Dioxetanes as mechanoluminescent probes in thermoplastic elastomers. Macromolecules 47, 3797-3805.

Chen, Y., Spiering, A.J.H., Karthikeyan, S., Peters, G.W.M., Meijer, E.W., Sijbesma, R.P., 2012. Mechanically induced chemiluminescence from polymers incorporating a 1,2-dioxetane unit in the main chain. Nature Chemistry 4, 559-562.

Cheng, L., Torres, Y., Min Lee, K., McClung, A.J., Baur, J., White, T.J., Oates, W.S., 2012. Photomechanical bending mechanics of polydomain azobenzene liquid crystal polymer network films. Journal of Applied Physics 112, 013513

Ciardelli, F., Ruggeri, G., Pucci, A., 2013. Dye-containing polymers: methods for preparation of mechanochromic materials. Chemical Society Reviews 42, 857-870.

Cohen Stuart, M.A., Huck, W.T.S., Genzer, J., Mller, M., Ober, C., Stamm, M., Sukhorukov, G.B., Szleifer, I., Tsukruk, V.V., Urban, M., Winnik, F., Zauscher, S., Luzinov, I., Minko, S., 2010. Emerging applications of stimuli-responsive polymer materials. Nature Materials 9, 101-113.

Crenshaw, B.R., Burnworth, M., Khariwala, D., Hiltner, A., Mather, P.T., Simha, R., Weder, C., 2007. Deformation-induced color changes in mechanochromic polyethylene blends. Macromolecules 40, 2400-2408.

Crenshaw, B.R., Weder, C., 2006. Self-assessing photoluminescent polyurethanes. Macromolecules 39, 9581-9589.

Davis, D.A., Hamilton, A., Yang, J., Cremar, L.D., Van Gough, D., Potisek, S.L., Ong, M.T., Braun, P.V., Martnez, T.J., White, S.R., Moore, J.S., Sottos, N., 2009. Force-induced activation of covalent bonds in mechanoresponsive polymeric materials. Nature 459, 68-72.

Debashish, R., Cambre, J.N., Sumerlin, B.S., 2010. Future perspectives and recent advances in stimuli-responsive materials. Progress in Polymer Science 35, 278-301.

Deng, H., Pence, T.J., 2010. Equilibrium states of mechanically loaded saturated and unsaturated polymer gels. Journal of Elasticity 99, 39-73.

Desai, S., Thakore, I., Sarawade, B., Devi, S., 2000. Effect of polyols and diisocyanates on thermo-mechanical and morphological properties of polyurethanes. European Polymer Journal 36, 711-725.

Donati, F., Pucci, A., Cappelli, C., Mennucci, B., Ruggeri, G., 2008. Modulation of the optical response of polyethylene films containing luminescent perylene chromophores. Journal of Physical Chemistry B 112, 3668-3679. 
Feng, F., Ye, L., 2011. Morphologies and mechanical properties of polylactide/thermoplastic polyurethane elastomer blends. Journal of Applied Polymer Science 119, 2778-2783.

Ferrari, M., Bashir, R., Wereley, S.T. (Eds.), 2007. BioMEMS and Biomedical Nanotechnology. Volume IV: Biomolecular Sensing, Processing and Analysis. Springer.

Foulger, S.H., Jiang, P., Ying, Y., Lattam, A.C., Smith, D.W., Ballato, J., 2001. Photonic bandgap composites. Advanced Materials 13, 1898-1901.

Gambarotta, L., Massabo, R., Morbiducci, R., Raposio, E., Santi, P., 2005. In vivo experimental testing and model identification of human scalp skin. Journal of Biomechanics 38, $2237-2247$.

Gossweiler, G.R., Hewage, G.B., Soriano, G., Wang, Q., Welshofer, G.W., Zhao, X., Craig, S.L., 2014. Mechanochemical activation of covalent bonds in polymers with full and repeatable macroscopic shape recovery. ACS Macro Letters 3, 216219.

Goulbourne, N., Mockensturm, E., Frecker, M., 2005. A nonlinear model for dielectric elastomer membranes. Journal of Applied Mechanics 72, 899-906.

Govindjee, S., Simo, J., 1991. A micro-mechanically based continuum damage model for carbon black-filled rubbers incorporating mullins' effect. Journal of the Mechanics and Physics of Solids 39, $87-112$.

Gupta, N., Pinisetty, D., Shunmugasamy, V.C., 2013. Reinforced Polymer Matrix Syntactic Foams. Springer.

Guth, E., 1945. Thory of filler reinforcement. Journal of Applied Physics 12, 20-25.

Harrison, J.S., Ounaies, Z., 2002. Piezoelectric Polymers. John Wiley \& Sons.

Hetch, E., 2002. Optics. Pearson.

Horgan, C.O., Ogden, R.W., Saccomandi, G., 2004. A theory of stress softening of elastomers based on finite chain extensibility. Proceedings of the Royal Society of London A: Mathematical, Physical and Engineering Sciences 460, $1737-1754$.

Jedynak, R., 2014. Approximation of the inverse langevin function revisited. Rheologica Acta , 1-11.

Jo, C., Pugal, D., Oh, I.K., Kim, K.J., Asaka, K., 2013. Recent advances in ionic polymer-metal composite actuators and their modeling and applications. Progress in Polymer Science 38, 1037-1066.

Kingsbury, C.M., May, P.A., Davis, D.A., White, S.R., Mooreabd, J.S., Sottos, N.R., 2011. Shear activation of mechanophorecrosslinked polymers. Journal of Materials Chemistry 21, 8381-8388.

Kunzelman, J., Chung, T., Mather, P.T., Weder, C., 2008. Shape memory polymers with built-in threshold temperature sensors. Journal of Materials Chemistry 18, 1082-1086.

Kunzelman, J., Crenshaw, B.R., Kinami, M., Weder, C., 2006. Self-assembly and dispersion of chromogenic molecules: A versatile and general approach for self-assessing polymers. Macromolecular Rapid Communications 27, $1981-1987$.

Lacarbonara, W., 2013. Nonlinear Structural Mechanics. Springer.

Larsen, M.B., Boydston, A.J., 2014. Successive mechanochemical activation and small molecule release in an elastomeric material. Journal of the American Chemical Society 136, 1276-1279.

Love, A.E.H., 1906. A treatise on the mathematical theory of elasticity. Cambridge University Press, London.

Makowski, B., Kunzelman, J., Weder, C., 2011. Stimuli-Driven Assembly of Chromogenic Dye Molecules: a Versatile Approach for the Design of Responsive Polymers. Wiley-VCH Verlag GmbH. pp. 117-138.

May, P.A., Moore, J.S., 2013. Polymer mechanochemistry: techniques to generate molecular force via elongational flows. Chemical Society Reviews 42, 7497-7506.

Pei, A., Malho, J.M., Ruokolainen, J., Zhou, Q., Berglund, L.A., 2011. Strong nanocomposite reinforcement effects in polyurethane elastomer with low volume fraction of cellulose nanocrystals. Macromolecules 44, 4422-4427.

Petrovic, Z.S., Ferguson, J., 1991. Polyurethane elastomers. Progress in Polymer Science 16, 695-836.

Petrovic, Z.S., Javni, I., Divjakovic, V., 1998. Structure and physical properties of segmented polyurethane elastomers containing chemical crosslinks in the hard segment. Journal of Polymer Science Part B Polymer Physics 36, 221-235.

Pucci, A., Bertoldo, M., Bronco, S., 2005. Luminescent bis(benzoxazolyl)stilbene as a molecular probe for poly(propylene) film deformation. Macromolecular Rapid Communications 26, 1043-1048.

Pucci, A., Bizzarri, R., Ruggeri, G., 2011. Polymer composites with smart optical properties. Soft Matter 7, 3689-3700.

Pucci, A., Cappelli, C., Bronco, S., Ruggeri, G., 2006. Dichroic properties of bis(benzoxazolyl)stilbene and bis(benzoxazolyl)thiophene dispersed into oriented polyethylene films: A combined experimental and density functional theory approach. The Journal of Physical Chemistry B 110, 3127-3134.

Pucci, A., Di Cuia, F., Signori, F., Ruggeri, G., 2007. Bis(benzoxazolyl)stilbene excimers as temperature and deformation sensors for biodegradable poly(1,4-butylene succinate) films. Journal of Materials Chemistry 17, 783-790.

Pucci, A., Ruggeri, G., 2011. Mechanochromic polymer blends. Journal of Materials Chemistry 21, 8282-8291.

Qi, H.J., Boyce, M.C., 2004. Constitutive model for stretch-induced softening of the stressstretch behavior of elastomeric materials. Journal of the Mechanics and Physics of Solids 52, 2187-2205.

Qi, H.J., Boyce, M.C., 2005. Stress-strain behavior of thermoplastic polyurethane. Mechanics of Materials 37, 817-839.

Rachik, M., Schmidtt, F., Reuge, N., Le Maoult, Y., Abbe, F., 2001. Elastomer biaxial characterization using bubble inflation technique. ii: Numerical investigation of some constitutive models. Polymer Engineering \& Science 41, 532-541.

Rivlin, R.S., Saunders, D.W., 1951. Large elastic deformations of isotropic materials. vii. experiments on the deformation of rubber. Philosophical Transactions of the Royal Society of London A 243, 251-288.

Rizzi, E., Papa, E., Corigliano, A., 2000. Mechanical behavior of a syntactic foam: experiments and modeling. International Journal of Solids and Structures 37, 5773-5794.

Roberts, D.R.T., Holder, S.J., 2011. Mechanochromic systems for the detection of stress, strain and deformation in polymeric materials. Journal of Materials Chemistry 21, 8256-8268.

Selvadurai, A., Shi, M., 2012. Fluid pressure loading of a hyperelastic membrane. International Journal of Non-Linear Mechanics 47, 228-239. 
Shundo, A., Okada, Y., Ito, F., Tanaka, K., 2012. Fluorescence behavior of dyes in thin films of various polymers. Macromolecules 45, 329-335.

Sottos, N.R., 2014. Polymer mechanochemistry: Flex, release and repeat. Nature Chemistry 6, 381-383.

Stokes, K., McVenes, R., Anderson, J.M., 1995. Polyurethane elastomer biostability. Journal of Biomaterials Applications 9, $321-354$

Thiyagasundaram, P., Sankar, B.V., Arakere, N.K., 2010. Elastic properties of open-cell foams with tetrakaidecahedral cells using finite element analysis. AIAA Journal 48, 818-828.

Urban, M.W. (Ed.), 2011. Handbook of Stimuli-Responsive Materials,. Wiley-V.C.H. Verlag, Weinheim, Germany.

Venugopal, V., Zhang, H., Northcutt, R., Sundaresan, V.B., 2014. A thermodynamic chemomechanical constitutive model for conducting polymers. Sensors and Actuators B: Chemical 201, $293-299$.

Wallmersperger, T., Attaran, A., Keller, K., Brummund, J., Guenther, M., Gerlach, G., 2013. Modeling and simulation of hydrogels for the application as bending actuators, in: Intelligent Hydrogels. Springer International Publishing.

Wang, Z., Pinnavaia, T.J., 1998. Nanolayer reinforcement of elastomeric polyurethane. Chemistry of Materials $10,3769-3771$.

Weder, C., 2011. Mechanoresponsive materials. Journal of Materials Chemistry 21, 8235-8236.

Yang, W.H., Feng, W.W., 1970. On axisymmetrical deformation of nonlinear membranes. Journal of Applied Mechanics 37, 1002-1011. 

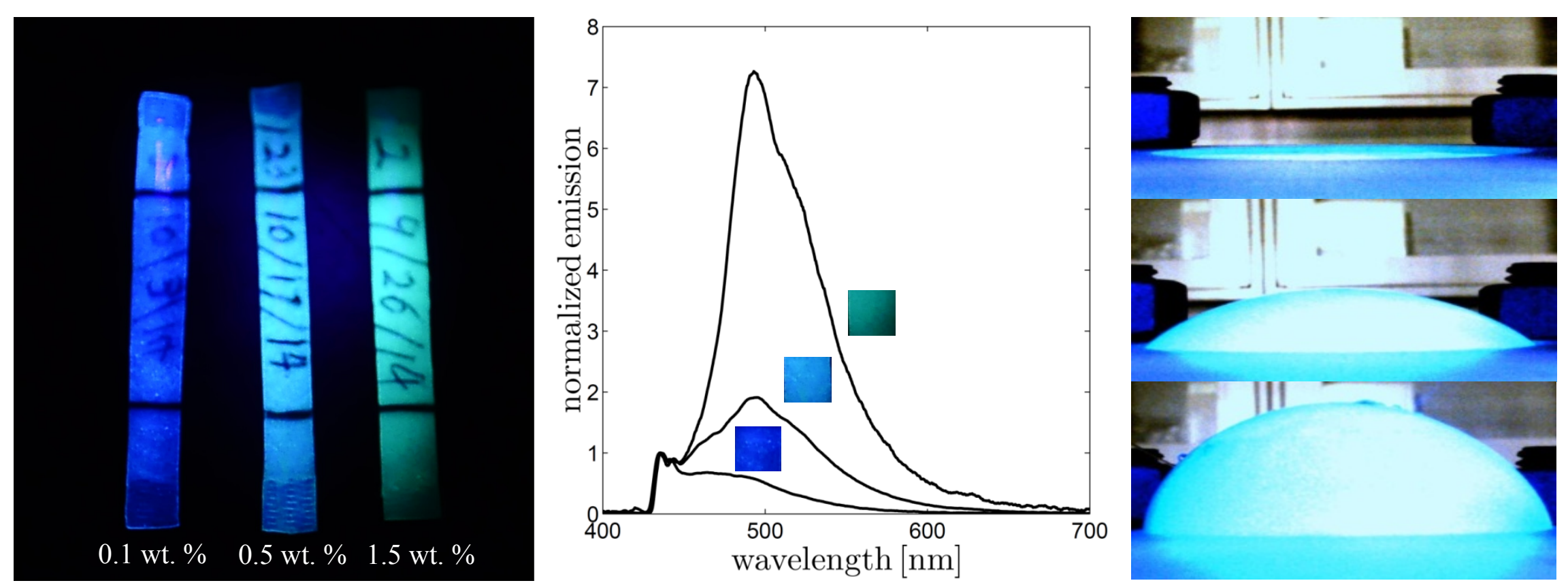